Proceedings of the Edinburgh Mathematical Society (2006) 49, 145-172 (C)

DOI:10.1017/S0013091504000446 Printed in the United Kingdom

\title{
LARGE-TIME BEHAVIOUR OF THE SOLUTIONS FOR A MULTIDIMENSIONAL NON-ISENTROPIC HYDRODYNAMIC MODEL FOR SEMICONDUCTORS
}

\author{
YEPING LI \\ Department of Mathematics, Xianning College, Xianning, People's Republic of China, \\ and Department of Mathematics, Shanghai University, Shanghai, \\ People's Republic of China (yepinglee197211@yahoo.com.cn)
}

(Received 4 June 2004)

\begin{abstract}
We investigate the multidimensional non-isentropic Euler-Poisson (or full hydrodynamic) model for semiconductors, which contain an energy-conserved equation with non-zero thermal conductivity coefficient. We first discuss existence and uniqueness of the non-constant stationary solutions to the corresponding drift-diffusion equations. Then we establish the global existence of smooth solutions to the Cauchy problem with initial data, which are close to the stationary solutions. We find that these smooth solutions tend to the stationary solutions exponentially fast as $t \rightarrow+\infty$.
\end{abstract}

Keywords: large-time behaviour; smooth solutions; multidimensional; non-isentropic hydrodynamic model; semiconductors

2000 Mathematics subject classification: Primary 35L65; 76X05; 35M10; 35L70; 35Q72

\section{Introduction}

In recent years there has been an increasing interest in the use of hydrodynamic-type models versus classical drift-diffusion models [22]. This is due to the need to model ever smaller devices and therefore the analysis obtained by the classical drift-diffusion models has previously been quite inaccurate. The relation between the drift-diffusion model and the classical isentropic hydrodynamic model was then developed in [20] and later investigated in [11]. However, the computational complexity of the hydrodynamic models was still forcing the use of drift-diffusion type modelling, hence the investigation of more realistic situations need to use a generalized version of the drift-diffusion models, namely the so-called 'energy transport' models. These new models were derived from extended thermodynamics in $[\mathbf{4}-\mathbf{6}]$ and other references quoted therein. There was a parallel analysis in $[\mathbf{8}, \mathbf{1 7}]$ which deduced essentially the same energy transport models. The paper by Ali et al. [2] presented the correct asymptotics to deduce the energy transport from the full hydrodynamic models. In this paper, we are interested in a multidimensional non-isentropic hydrodynamic model for semiconductors. After appropriate 
scaling, our model equations are given by

$$
\left.\begin{array}{rl}
n_{t}+\nabla \cdot(n \boldsymbol{u}) & =0, \\
\boldsymbol{u}_{t}+(\boldsymbol{u} \cdot \nabla) \boldsymbol{u}+\frac{1}{n} \nabla(n T) & =\nabla \Phi-\frac{\boldsymbol{u}}{\tau_{1}}, \\
T_{t}+\boldsymbol{u} \cdot \nabla T+\frac{2}{3} T \nabla \cdot \boldsymbol{u}-\frac{2}{3 n} \nabla \cdot(\kappa \nabla T) & =\frac{2 \tau_{2}-\tau_{1}}{3 \tau_{1} \tau_{2}}|\boldsymbol{u}|^{2}-\frac{T-T^{(0)}}{\tau_{2}}, \\
\Delta \Phi & =n-b(x),
\end{array}\right\}
$$

for $(x, t) \in \mathbb{R}^{d} \times[0,+\infty), d=2,3$, here $n, \boldsymbol{u}, \Phi$ and $T$ denote the electron density, the electron velocity, the electrostatic potential and the carrier temperature, respectively. The coefficients $\kappa, \tau_{p}$ and $\tau_{w}$ are thermal conductivity coefficient, the momentum relaxation time and energy relaxation time, respectively. In general, the physical constants may depend on $n$ and $T$. In this paper, we only discuss the case when $\tau_{1}, \tau_{2}$ and $\kappa$ are constant. The positive constant $T^{(0)}$ is the ambient device temperature. The function $b(x)$ denotes the prescribed density of positively charged background ions (doping profile). Often the energy Equation $(1.1)_{3}$ is replaced by a pressure-density relation $p(n)=k n^{r}, k>0$, $r \geqslant 1$; the corresponding model is referred to as the unipolar isentropic hydrodynamic model for semiconductors. Furthermore, if there are both electrons and holes present, we regard the model as the bipolar isentropic (or non-isentropic) hydrodynamic model for semiconductors. For more discussion on these models in physics and engineering, and their derivation from kinetic transport models by the moment method, we refer the reader to $[\mathbf{1 7}, \mathbf{2 2}]$.

For simplicity, we can assume that the constants $\kappa, \tau_{1}$ and $\tau_{2}$ are 1 , but this does not affect the subsequent analysis. Moreover, introducing the electric field $e$ by $e=\nabla \Phi$, we can rewrite (1.1) as

$$
\left.\begin{array}{rl}
n_{t}+\nabla \cdot(n \boldsymbol{u}) & =0, \\
\boldsymbol{u}_{t}+(\boldsymbol{u} \cdot \nabla) \boldsymbol{u}+\frac{1}{n} \nabla(n T) & =e-\boldsymbol{u}, \\
T_{t}+\boldsymbol{u} \cdot \nabla T+\frac{2}{3} T \nabla \cdot \boldsymbol{u}-\frac{2}{3 n}(\Delta T) & =\frac{1}{3}|\boldsymbol{u}|^{2}-\left(T-T^{(0)}\right), \\
\nabla \cdot e & =n-b(x) .
\end{array}\right\}
$$

We consider the Cauchy problem of (1.2) in this paper, and supplement (1.2) with the following initial data:

$$
(n(x, 0), \boldsymbol{u}(x, 0), T(x, 0))=\left(n_{0}(x), \boldsymbol{u}_{0}(x), T_{0}(x)\right), \quad x \in \mathbb{R}^{d}, \quad d=2,3 .
$$

Using Green's formulation, it follows from (1.2) and (1.3) that

$$
e(x, t)=\nabla \Phi=\nabla \Delta^{-1}\left(n_{0}-b(x)\right)-\nabla \Delta^{-1} \nabla \cdot \int_{0}^{t}(n u)(x, s) \mathrm{d} s .
$$


Moreover, our assumptions on $b(x)$ are

$$
\lim _{|x| \rightarrow \infty} b(x)=\bar{b}>0, \quad b(x)>0, \quad 0<\inf _{x \in \mathbb{R}^{d}} b(x) \leqslant b(x) \leqslant \sup _{x \in \mathbb{R}^{d}} b(x),
$$

and

$$
b(x) \in C^{6}\left(\mathbb{R}^{d}\right), \quad \nabla b \in H^{5}\left(\mathbb{R}^{d}\right), \quad d=2,3 .
$$

It is expected that the solutions of (1.2) and (1.3) tend to some stationary solutions as $t \rightarrow+\infty$. Let us now turn our attention to considering the steady-state drift-diffusion equation when the velocity $\boldsymbol{u}=\mathbf{0}$ (the total thermodynamic equilibrium steady state). We can investigate the stationary solution $(N, E)$ of the system

$$
\left.\begin{array}{rl}
T^{(0)} \nabla N & =N E, \\
\nabla \cdot E & =N-b(x),
\end{array}\right\}
$$

under the assumption of

$$
N-b(x) \in H^{5}\left(\mathbb{R}^{d}\right) .
$$

Since Degond and Markowich started a mathematical analysis for the simplified steady-state hydrodynamic model in [7], hydrodynamic models for semiconductors have attracted a lot of attention because of their ability to model hot-electron effects that are not accounted for in the classical drift-diffusion model. Some topics such as the steady-state solutions $[\mathbf{7}, \mathbf{8}, \mathbf{1 0}, \mathbf{2 1}]$, weak solutions $[\mathbf{9}, \mathbf{1 6}, \mathbf{2 0}, \mathbf{2 5}, \mathbf{2 7}]$, and the relaxation relation between the hydrodynamic model and the drift-diffusion model $[\mathbf{1 6}, \mathbf{2 0}]$, have been studied extensively. In particular, after Luo et al. [18] first investigated the global solutions and the asymptotic behaviour of the smooth solution to the Cauchy problem for the one-dimensional isentropic hydrodynamic model, Hsiao and Yang [14] discussed the corresponding initial-boundary-value problem, Markowich et al. [15] investigated the multidimensional isentropic hydrodynamic model case, and Guo [12] studied the multidimensional isentropic irrotational case. Furthermore, Hsiao and Wang [13] discussed the large-time behaviour of the solutions for the one-dimensional non-isentropic EulerPoisson equation for semiconductors, while Ali et al. $[\mathbf{3}]$ and Ani $[\mathbf{1}]$ investigated the onedimensional and the multidimensional non-isentropic cases with the zero-thermoductivity coefficient. It is more interesting to study the multidimensional general hydrodynamic system for semiconductors, but it is very difficult to establish the global existence of weak or smooth solutions. This paper addresses the important question of the asymptotic stability of the steady states for the hydrodynamic models. This question is important because the steady states of the full hydrodynamic models coincide with those of the classical drift-diffusion model. Therefore, by proving the asymptotic stability, we can conclude that the classical drift-diffusion model can replace the full hydrodynamic model when the initial data are close to the steady regime. This is the purpose of the present paper. This task is achieved by means of standard energy-type methods, but with severe restrictions that are not completely reasonable from the point of view of physics. For example, $\|\nabla b(x)\|_{H^{5}\left(\mathbb{R}^{d}\right)}$ is sufficiently small that we can prove the existence of a steady-state solution for (1.2) and (1.3) and establish the large-time behaviour of 
smooth solutions for (1.2) and (1.3). We now state our main results as follows. We shall first prove the existence and uniqueness result for (1.7) and (1.8).

Theorem 1.1. Let $b(x)$ satisfy equations (1.5) and (1.6). Moreover, we assume that $\|\nabla b(x)\|_{H^{5}\left(\mathbb{R}^{d}\right)}$ is sufficiently small. There then exists a unique pair $(N, E)$, which is a solution of (1.7) and (1.8).

We can then establish the global existence and the large-time behaviour of the global smooth solution to the Cauchy problem for (1.2) and (1.3).

Theorem 1.2. Let $(N, E)$ be the solution of (1.7) and (1.8). Assume that $b(x)$ satisfies (1.5) and (1.6), and $\left(n_{0}-N, \boldsymbol{u}_{0}, e_{0}-E\right) \in H^{3}\left(\mathbb{R}^{d}\right), T_{0}-T^{(0)} \in H^{4}\left(\mathbb{R}^{d}\right)$. Then there exists a positive number $\delta$ such that if

$$
\begin{aligned}
\left\|\left(n_{0}-N, \boldsymbol{u}_{0}, e_{0}-E\right)\right\|_{H^{3}\left(\mathbb{R}^{d}\right)}+\left\|T_{0}-T^{(0)}\right\|_{H^{4}\left(\mathbb{R}^{d}\right)} \\
+\left\|\left(n_{t}, \boldsymbol{u}_{t}, e_{t}, T_{t}\right)(x, 0)\right\|_{H^{2}\left(\mathbb{R}^{d}\right)}+\|\nabla b(x)\|_{H^{5}\left(\mathbb{R}^{d}\right)} \leqslant \delta,
\end{aligned}
$$

then the Cauchy problem (1.2), (1.3) admits a unique smooth solution $(n, \boldsymbol{u}, e, T)$ for all $t>0$. Moreover,

$$
\begin{gathered}
\|(n-N, \boldsymbol{u}, e-E)\|_{H^{3}\left(\mathbb{R}^{d}\right)}^{2}+\left\|T-T^{(0)}\right\|_{H^{4}\left(\mathbb{R}^{d}\right)}^{2}+\left\|\left(n_{t}, \boldsymbol{u}_{t}, e_{t}, T_{t}\right)\right\|_{H^{2}\left(\mathbb{R}^{d}\right)}^{2} \\
\leqslant C\left(\left\|\left(n_{0}-N, \boldsymbol{u}_{0}, e_{0}-E\right)\right\|_{H^{3}\left(\mathbb{R}^{d}\right)}^{2}+\left\|T_{0}-T^{(0)}\right\|_{H^{4}\left(\mathbb{R}^{d}\right)}^{2}\right. \\
\left.+\left\|\left(n_{t}, \boldsymbol{u}_{t}, e_{t}, T_{t}\right)(x, 0)\right\|_{H^{2}\left(\mathbb{R}^{d}\right)}^{2}\right) \exp (-\alpha t),
\end{gathered}
$$

for some positive constants $\alpha$ and $C$, where $\left(n_{t}, \boldsymbol{u}_{t}, e_{t}, T_{t}\right)(x, 0)$ is defined through (1.2)-(1.4).

Remark 1.3. In contrast to $[\mathbf{1 3}]$, we establish the non-constant stationary solutions; consequently, we need to make more careful estimates in order to overcome more difficulties caused by those terms arising from the non-constant steady-state solutions and by the more general multi-dimensional case $d=2,3$. Meanwhile, we discuss the more general hydrodynamic model for semiconductors than the model which is indeed a hyperbolicelliptic system with zero thermal conductivity coefficient in [1] , because it is a hyperbolicelliptic-parabolic coupled system.

\subsection{Notation}

$H^{s}\left(\mathbb{R}^{d}\right), d \in \mathbb{Z}_{+}$denotes the usual Sobolev space of order $s$ equipped with the norm

$$
\|g\|_{H^{s}\left(\mathbb{R}^{d}\right)}=\sum_{0 \leqslant|\alpha| \leqslant s}\left\|\partial_{x}^{\alpha} g\right\|
$$

where $\|\cdot\|=\|\cdot\|_{L^{2}\left(\mathbb{R}^{d}\right)}$ and $\partial_{x}^{\alpha}=\partial_{1}^{\alpha_{1}} \partial_{2}^{\alpha_{2}} \cdots \partial_{d}^{\alpha_{d}}$ with $\sum_{i=1}^{d} \alpha_{i}=\alpha$ and $\partial_{i}=\partial_{x_{i}}$. The Euclidean norm and inner product for $\mathbb{R}^{d}$ are denoted by $|\cdot|$ and $a \cdot b$ for $a, b \in \mathbb{R}^{d}$, respectively. For a vector-valued function $f=\left(f_{1}, f_{2}, \ldots, f_{k}\right)$ and a normed space $X$, 
scalar functions with the norm $\|\cdot\| \|(f \in X$ means that each component of $f$ is in $X)$, we put

$$
\|f\||=|\left\|f_{1}\right\|\left|+\left\|f_{2}\left|\|+\cdots+\| f_{k} \|\right|\right.\right.
$$

and

$$
\partial f=\partial_{x} f=\left(\partial_{i} f_{j}\right)_{d \times k}, \quad \partial_{x}^{k} f=\partial_{x}\left(\partial_{x}^{k-1} f\right) .
$$

$C$ always stands for a generic constant, $C(\cdot)$ means that $C$ depends on ' ', and $\varepsilon$ denotes an arbitrary positive constant which comes from the Young inequality. Repeated indices mean summation from 1 to $d$. $\int \cdot \mathrm{d} x$ always denotes $\int_{\mathbb{R}^{d}} \cdot \mathrm{d} x$.

Moreover, we also give two useful inequalities which are used repeatedly in this note.

\subsubsection{Young's inequality}

Let $a, b, \varepsilon$ be positive constants an $p_{0}, q_{0} \geqslant 1,1 / p_{0}+1 / q_{0}=1$. Then

$$
a b \leqslant \frac{\varepsilon^{p_{0}} a^{p_{0}}}{p_{0}}+\frac{b^{q_{0}}}{\varepsilon^{q_{0}} q_{0}} .
$$

When $p_{0}+q_{0}=2$, we have the Cauchy-Schwartz inequality

$$
|a b| \leqslant \varepsilon a^{2}+\frac{b^{2}}{4 \varepsilon} .
$$

1.1.2. Moser-type calculus [1]

If $f, g \in H^{k} \cap L^{\infty}$, then we have

$$
\left\|D^{k}(f g)\right\| \leqslant C(k)\left(\left.\left\|\left.f\right|_{L^{\infty}}\right\| D^{k} g\|+\| g\right|_{L^{\infty}}\left\|D^{k} f\right\|\right) .
$$

1.1.3. Gagliardo-Nirenberg inequality [24]

$$
\|u\|_{L^{q}} \leqslant C(d, q)\|u\|^{d / q-d / 2+1}\|D u\|^{d / 2-d / q},
$$

for $u \in H^{1}\left(\mathbb{R}^{d}\right), q \geqslant 2$ when $d=2$ and $q \in[2,6]$ when $d=3$, while for $u \in H^{2}\left(\mathbb{R}^{d}\right)$

$$
\|u\|_{L^{\infty}} \leqslant C(d)\|u\|^{(4-d) / 4}\left\|D^{2} u\right\|^{d / 4} .
$$

This paper is arranged as follows. We first investigate the existence and uniqueness of the stationary solutions in $\S 2$. Section 3 is devoted to the global existence and large-time behaviour of smooth solutions for the multidimensional non-isentropic hydrodynamic model for semiconductors.

\section{The stationary solution of the drift-diffusion equation}

In this section, we shall prove Theorem 1.1. The proof is based on the crucial a priori estimates given in the following lemma. 
Lemma 2.1. Let $b(x)$ satisfy (1.5) and (1.6), and let $(N, E)$ be a solution of (1.7) and (1.8) given by Theorem 1.1. Moreover, assume that $\|\nabla b(x)\|_{H^{5}\left(\mathbb{R}^{d}\right)}$ is sufficiently small. Then the following estimates hold:

$$
\begin{aligned}
\inf _{x \in \mathbb{R}^{d}} b(x) & \leqslant N(x) \leqslant \sup _{x \in \mathbb{R}^{d}} b(x), \\
\|\nabla N\|_{H^{5}\left(\mathbb{R}^{d}\right)}^{2} & \leqslant C\left(\|\nabla b(x)\|_{H^{5}\left(\mathbb{R}^{d}\right)}^{2}\right) .
\end{aligned}
$$

Proof. The system (1.7) is equivalent to the nonlinear equation

$$
T^{(0)} \Delta \ln N=N-b(x) .
$$

Since $\lim _{|x| \rightarrow \infty} N(x)=\lim _{|x| \rightarrow \infty} b(x)=\bar{b}$, from the maximum principle we immediately find that

$$
0<\inf _{x \in \mathbb{R}^{d}} b(x) \leqslant N(x) \leqslant \sup _{x \in \mathbb{R}^{d}} b(x) .
$$

Now we derive the a priori estimate (2.2). We differentiate (2.3) with respect to $x$ and multiply the resultant equation by $\nabla \ln N$. Integration over $\mathbb{R}^{d}$ leads to

$$
\int T^{(0)} \nabla \Delta \ln N \nabla \ln N \mathrm{~d} x=\int \nabla(N-b(x)) \nabla \ln N \mathrm{~d} x ;
$$

using the Cauchy-Schwartz inequality and integration by parts, we discover that

$$
\int\left(\left|\nabla^{2} \ln N\right|^{2}+|\nabla(N-b(x))|^{2}\right) \mathrm{d} x \leqslant C \int|\nabla b(x)|^{2} \mathrm{~d} x .
$$

Taking $\nabla^{2}$ in (2.3), multiplying the resultant equation by $\nabla^{2} \ln N$ and integrating it over $\mathbb{R}^{d}$, we see that

$$
\int T^{(0)} \nabla^{2} \Delta \ln N \nabla^{2} \ln N \mathrm{~d} x=\int \nabla^{2}(N-b(x)) \nabla^{2} \ln N \mathrm{~d} x,
$$

which yields

$$
\int\left(\left|\nabla^{3} \ln N\right|^{2}+\left|\nabla^{2}(N-b(x))\right|^{2}\right) \mathrm{d} x \leqslant C \int\left(\left|\nabla^{2} b(x)\right|^{2}+|\nabla N|^{4}\right) \mathrm{d} x .
$$

Moreover, using (1.11)-(1.14), we get

$$
\begin{aligned}
\int|\nabla N|^{4} \mathrm{~d} x & =\int\left|\frac{\nabla \ln N}{\ln ^{\prime} N}\right|^{4} \mathrm{~d} x \\
& \leqslant C \int|\nabla \ln N|^{4} \mathrm{~d} x \\
& \leqslant \varepsilon \int\left|\nabla^{3} \ln N\right|^{2} \mathrm{~d} x+C(\varepsilon)\left(\int|\nabla \ln N|^{2} \mathrm{~d} x\right)^{(8-d) /(4-d)} .
\end{aligned}
$$


By inserting (2.6) into (2.5) we can deduce that

$$
\int\left(\left|\nabla^{3} \ln N\right|^{2}+\left|\nabla^{2}(N-b(x))\right|^{2}\right) \mathrm{d} x \leqslant C\left(\int\left|\nabla^{2} b(x)\right|^{2} \mathrm{~d} x+\left(\int|\nabla \ln N|^{2} \mathrm{~d} x\right)^{(8-d) /(4-d)}\right) .
$$

We then turn our attention to the higher-derivative estimates. By substituting $\nabla^{3}$ in (2.3), multiplying the resultant equation by $\nabla^{3} \ln N$ and integrating it over $\mathbb{R}^{d}$, we find that

$$
\int T^{(0)} \nabla^{3} \Delta \ln N \nabla^{3} \ln N \mathrm{~d} x=\int \nabla^{3}(N-b(x)) \nabla^{3} \ln N \mathrm{~d} x,
$$

which implies

$$
\begin{aligned}
& \int\left(\left|\nabla^{4} \ln N\right|^{2}+\left|\nabla^{3}(N-b(x))\right|^{2}\right) \mathrm{d} x \\
& \leqslant C\left(\int\left|\nabla^{3} b(x)\right|^{2} \mathrm{~d} x+\int\right.\left(|\nabla(N-b(x))+\nabla b(x)|^{6}+|\nabla(N-b(x))|^{2}\left|\nabla^{2}(N-b(x))\right|^{2}\right. \\
&+|\nabla(N-b(x))|^{2}\left|\nabla^{2} b(x)\right|^{2}+|\nabla b(x)|^{2}\left|\nabla^{2}(N-b(x))\right|^{2} \\
&\left.\left.+|\nabla b(x)|^{2}\left|\nabla^{2} b(x)\right|^{2}\right) \mathrm{~d} x\right) .
\end{aligned}
$$

Now let us control the second integral term in the right-hand side of the above inequality. Using (1.11)-(1.14), we can conclude that

$$
\begin{aligned}
\int \mid \nabla( & N-b(x))+\left.\nabla b(x)\right|^{6} \mathrm{~d} x \\
& \leqslant C \int|\nabla \ln N|^{6} \mathrm{~d} x \leqslant C \sup _{x \in \mathbb{R}^{d}}|\nabla \ln N|^{4} \int|\nabla \ln N|^{2} \mathrm{~d} x \\
& \leqslant C\left(\int\left|\nabla^{2} b(x)\right|^{2} \mathrm{~d} x+\left(\int|\nabla b(x)|^{2} \mathrm{~d} x\right)^{(8-d) /(4-d)}\right)^{d / 2}\left(\int|\nabla b(x)|^{2} \mathrm{~d} x\right)^{(6-d) / 2}
\end{aligned}
$$

and

$$
\begin{aligned}
& \int|\nabla(N-b(x))|^{2}\left|\nabla^{2}(N-b(x))\right|^{2} \mathrm{~d} x \\
& \leqslant \sup _{x \in \mathbb{R}^{d}}|\nabla(N-b(x))|^{2} \int\left|\nabla^{2}(N-b(x))\right|^{2} \mathrm{~d} x \\
& \leqslant C\left(\int\left|\nabla^{3}(N-b(x))\right|^{2} \mathrm{~d} x\right)^{d / 4}\left(\int|\nabla(N-b(x))|^{2} \mathrm{~d} x\right)^{(4-d) / 4} \int\left|\nabla^{2}(N-b(x))\right|^{2} \mathrm{~d} x \\
& \leqslant \varepsilon \int\left|\nabla^{3}(N-b(x))\right|^{2} \mathrm{~d} x \\
& \quad+C(\varepsilon) \int|\nabla b(x)|^{2} \mathrm{~d} x\left(\int\left|\nabla^{2} b(x)\right|^{2} \mathrm{~d} x+\left(\int|\nabla b(x)|^{2} \mathrm{~d} x\right)^{(8-d) /(4-d)}\right)^{4 /(4-d)}
\end{aligned}
$$


Analogously, we can also prove that

$$
\begin{aligned}
& \int\left(|\nabla(N-b(x))|^{2}\left|\nabla^{2} b(x)\right|^{2}+|\nabla b(x)|^{2}\left|\nabla^{2}(N-b(x))\right|^{2}+|\nabla b(x)|^{2}\left|\nabla^{2} b(x)\right|^{2}\right) \mathrm{d} x \\
& \leqslant \varepsilon \int\left|\nabla^{3}(N-b(x))\right|^{2} \mathrm{~d} x \\
& +C(\varepsilon)\left(\int|\nabla b(x)|^{2} \mathrm{~d} x\left(\int\left|\nabla^{2} b(x)\right|^{2} \mathrm{~d} x\right)^{4 /(4-d)}\right. \\
& \left.\quad+\int|\nabla b(x)|^{2} \mathrm{~d} x\left(\int\left|\nabla^{2} b(x)\right|^{2} \mathrm{~d} x+\left(\int|\nabla b(x)|^{2} \mathrm{~d} x\right)^{(8-d) /(4-d)} \mathrm{d} x\right)^{4 /(4-d)}\right) \\
& +C(\varepsilon) \int\left|\nabla^{3} b(x)\right|^{2} \mathrm{~d} x .
\end{aligned}
$$

Therefore, noting that $\varepsilon$ is small enough, it follows that

$$
\begin{aligned}
& \int\left(\left|\nabla^{4} \ln N\right|^{2}+\left|\nabla^{3}(N-b(x))\right|^{2}\right) \mathrm{d} x \\
& \leqslant C \int\left|\nabla^{3} b(x)\right|^{2} \mathrm{~d} x \\
& +C\left(\int|\nabla b(x)|^{2} \mathrm{~d} x\left(\int\left|\nabla^{2} b(x)\right|^{2} \mathrm{~d} x\right)^{4 /(4-d)}\right. \\
& +\left(\int\left(|\nabla b(x)|^{2}+\left|\nabla^{2} b(x)\right|^{2}\right) \mathrm{d} x\right)^{d / 2}\left(\int|\nabla b(x)|^{2} \mathrm{~d} x\right)^{(6-d) / 2} \\
& \left.+\int|\nabla b(x)|^{2} \mathrm{~d} x\left(\int\left(|\nabla b(x)|^{2}+\left|\nabla^{2} b(x)\right|^{2}\right) \mathrm{d} x\right)^{4 /(4-d)}\right) .
\end{aligned}
$$

In a similar manner, we can find

$$
\int\left(\left|\nabla^{5} \ln N\right|^{2}+\left|\nabla^{6} \ln N\right|^{2}+\left|\nabla^{4}(N-b(x))\right|^{2}+\left|\nabla^{5}(N-b(x))\right|^{2}\right) \mathrm{d} x \leqslant C\left(\|\nabla b(x)\|_{H^{5}\left(\mathbb{R}^{d}\right)}\right) .
$$

Summing up (2.4) and (2.7)-(2.9), with the aid of (2.1), we can complete the proof of $(2.2)$.

Proof of Theorem 1.1. Based on Lemma 2.1, the standard iteration technique and fixed-point principle can be used to prove the existence of a stationary solution of (1.7) and (1.8) as in [18] (we omit the details). For the uniqueness of the steady-state solution, let us assume that both $\left(N_{1}, E_{1}\right)$ and $\left(N_{2}, E_{2}\right)$ satisfy $(1.7)$, and $N_{1}-b(x), N_{2}-b(x) \in$ $H^{5}\left(\mathbb{R}^{d}\right)$. Then, using Moser's calculus, $\ln \left(N_{1}\right)-\ln \left(N_{2}\right) \in H^{5}\left(\mathbb{R}^{d}\right)$. It follows that, for any $\delta_{0} \geqslant 0$, there exists $A \geqslant 0$ such that

$$
-\delta_{0} \leqslant \ln \left(N_{1}(x)\right)-\ln \left(N_{2}(x)\right) \leqslant \delta_{0}, \quad|x| \geqslant A .
$$


We will prove by contradiction that the inequalities in (2.10) also hold for all $|x| \leqslant A$. In fact, let us assume that

$$
\ln \left(N_{1}(\bar{x})\right)-\ln \left(N_{2}(\bar{x})\right) \equiv \max _{|x| \leqslant A}\left(\ln \left(N_{1}(x)\right)-\ln \left(N_{2}(x)\right)\right)>\delta_{0} .
$$

Then we find that

$$
\nabla\left(\ln \left(N_{1}(\bar{x})\right)-\ln \left(N_{2}(\bar{x})\right)\right)=0, \quad \Delta\left(\ln \left(N_{1}(\bar{x})\right)-\ln \left(N_{2}(\bar{x})\right)\right) \leqslant 0 .
$$

From (1.7), the two solutions, we find that

$$
\begin{aligned}
T^{(0)} \nabla\left(\ln \left(N_{1}(x)\right)-\ln \left(N_{2}(x)\right)\right)_{x} & =E_{1}(x)-E_{2}(x), \\
\operatorname{div}\left(E_{1}(x)-E_{2}(x)\right) & =N_{1}(x)-N_{2}(x),
\end{aligned}
$$

which implies that

$$
N_{1}(\bar{x})-N_{2}(\bar{x})=T^{(0)} \Delta\left(\ln \left(N_{1}(\bar{x})\right)-\ln \left(N_{2}(\bar{x})\right)\right) \leqslant 0 .
$$

Because $N>0$, we find that $\mathrm{d} / \mathrm{d} N \ln N>0$. Further, we end up with

$$
\ln \left(N_{1}(\bar{x})\right)-\ln \left(N_{2}(\bar{x})\right) \leqslant 0,
$$

which contradicts (2.11). Thus, the second inequality in (2.10) holds for all $x \in \mathbb{R}^{d}$. In the same way, we can prove that the first inequality in (2.10) is globally valid. In conclusion, we have proved that

$$
\left|\ln \left(N_{1}(x)\right)-\ln \left(N_{2}(x)\right)\right| \leqslant \delta_{0}, \quad x \in \mathbb{R}^{d} .
$$

It follows that $\ln \left(N_{1}\right)=\ln \left(N_{2}\right)$. Therefore, $N_{1}=N_{2}$ and $E_{1}=E_{2}$. This ends the proof.

\section{Global existence and large-time behaviour of the solutions for the non-isentropic hydrodynamic model}

In this section we shall prove Theorem 1.2. We investigate the global existence and largetime behaviour of the smooth solutions for $(1.2)$ and $(1.3)$. Let $(N, E)$ be the solution to (1.7) and (1.8), and introduce the following system:

$$
\left.\begin{array}{rl}
n & =N+m, \\
\boldsymbol{u} & =\boldsymbol{u}, \\
T & =T^{(0)}+y, \\
e & =E+\varphi .
\end{array}\right\}
$$


Then $(m, \boldsymbol{u}, y, \varphi)$ satisfies

$$
\left.\begin{array}{rl}
m_{t}+\nabla \cdot((N+m) \boldsymbol{u}) & =0, \\
\boldsymbol{u}_{t}+(\boldsymbol{u} \cdot \nabla) \boldsymbol{u}+\frac{T^{(0)}+y}{N+m} \nabla m+\frac{N y-T^{(0)} m}{N(N+m)} \nabla N+\nabla y & =\varphi-\boldsymbol{u}, \\
y_{t}+\boldsymbol{u} \cdot \nabla y+\frac{2}{3}\left(T^{(0)}+y\right) \nabla \cdot \boldsymbol{u}-\frac{2}{3(N+m)} \Delta y-\frac{1}{3}|\boldsymbol{u}|^{2}+y & =0, \\
\nabla \cdot \varphi & =m,
\end{array}\right\}
$$

and we have the corresponding initial data for $(m, \boldsymbol{u}, y, \varphi)$ :

$$
\left.\begin{array}{c}
(m(x, 0), \boldsymbol{u}(x, 0), y(x, 0))=\left(n_{0}-N, \boldsymbol{u}_{0}, T_{0}-T^{(0)}\right)=:\left(m_{0}, \boldsymbol{u}_{0}, y_{0}\right), \\
\varphi(x, 0)=e(x, 0)-E=\nabla \Delta^{-1}\left(n_{0}-b\right)-E=: \varphi_{0} .
\end{array}\right\}
$$

Therefore, the global existence and asymptotic behaviour for the Cauchy problem (1.2), (1.3) are reduced to the corresponding problem for (3.1), (3.2). Now, we can restate our main result on $(m, \boldsymbol{u}, y, \varphi)$ as follows.

Theorem 3.1. Assume that all the conditions in Theorem 1.2 are met. There then exists a unique smooth solution $(m, \boldsymbol{u}, y, \varphi)$ to (3.1) and (3.2) for all $t>0$. Moreover,

$$
\begin{aligned}
& \|(m, \boldsymbol{u}, \varphi)(\cdot, t)\|_{H^{3}\left(\mathbb{R}^{d}\right)}^{2}+\|y(\cdot, t)\|_{H^{2}\left(\mathbb{R}^{d}\right)}^{2}+\left\|\left(m_{t}, \boldsymbol{u}_{t}, y_{t}, \varphi_{t}\right)(\cdot, t)\right\|_{H^{2}\left(\mathbb{R}^{d}\right)}^{2} \\
& \quad \leqslant C\left(\left\|\left(m_{0}, \boldsymbol{u}_{0}, \varphi_{0}\right)\right\|_{H^{3}\left(\mathbb{R}^{d}\right)}^{2}+\left\|y_{0}\right\|_{H^{2}\left(\mathbb{R}^{d}\right)}^{2}+\left\|\left(m_{t}, \boldsymbol{u}_{t}, y_{t}, \varphi_{t}\right)(\cdot, 0)\right\|_{H^{2}\left(\mathbb{R}^{d}\right)}^{2}\right) \mathrm{e}^{-\alpha t}
\end{aligned}
$$

It is clear that Theorem 3.1 is equivalent to Theorem 1.2, so we only need to prove Theorem 3.1. Analogous to (1.4), we have

$$
\varphi=\nabla \Delta^{-1}\left(m_{0}\right)-\nabla \Delta^{-1} \nabla \cdot \int_{0}^{t}(N+m) \boldsymbol{u}(x, s) \mathrm{d} s .
$$

Since the non-local term $\nabla \Delta^{-1} \nabla \cdot \int_{0}^{t}(N+m) \boldsymbol{u}(x, s) \mathrm{d} s$ is the sum of the products of the Riesz transform of $\int_{0}^{t}(N+m) \boldsymbol{u}(x, s) \mathrm{d} s$, we find, by the $L^{2}$ boundedness of the Riesz transform $[\mathbf{2 6}]$, that

$$
\left\|\nabla \Delta^{-1} \nabla \cdot \int_{0}^{t}(N+m) \boldsymbol{u}(x, s) \mathrm{d} s\right\|_{H^{3}\left(\mathbb{R}^{d}\right)} \leqslant\left\|\int_{0}^{t}(N+m) \boldsymbol{u}(x, s) \mathrm{d} s\right\|_{H^{3}\left(\mathbb{R}^{d}\right)},
$$

for some constant $C>0$. Using this crucial fact, we can obtain the following local existence lemma from the symmetric hyperbolic system $[\mathbf{1 9}, \mathbf{2 3}]$.

Lemma 3.2. Assume that $\left(m_{0}, \boldsymbol{u}_{0}, \varphi_{0}\right) \in H^{3}\left(\mathbb{R}^{d}\right), y_{0} \in H^{4}\left(\mathbb{R}^{d}\right)$. There then exists a unique smooth solution

$$
\begin{gathered}
(m, \boldsymbol{u}, \varphi) \in C\left(\left[0, T_{\max }\right), H^{3}\left(\mathbb{R}^{d}\right)\right)^{3} \cap C^{1}\left(\left[0, T_{\max }\right), H^{2}\left(\mathbb{R}^{d}\right)\right)^{3}, \quad y \in C\left(\left[0, T_{\max }\right)\right), \\
H^{3}\left(\mathbb{R}^{d}\right) \cap C^{1}\left(\left[0, T_{\max }\right), H^{2}\left(\mathbb{R}^{d}\right)\right) \cap L^{2}\left(\left[0, T_{\max }\right), H^{4}\left(\mathbb{R}^{d}\right)\right)
\end{gathered}
$$


of (3.1) and (3.2), defined on a maximal interval existence [0, $\left.T_{\max }\right)$. Moreover, if $T_{\max }<$ $\infty$, then

$$
\begin{aligned}
& \|(m, \boldsymbol{u}, \varphi)(\cdot, t)\|_{H^{3}\left(\mathbb{R}^{d}\right)}^{2}+\|y(\cdot, t)\|_{H^{4}\left(\mathbb{R}^{d}\right)}^{2}+\left\|\left(m_{t}, \boldsymbol{u}_{t}, \varphi_{t}, y_{t}\right)(\cdot, t)\right\|_{H^{2}\left(\mathbb{R}^{d}\right)}^{2} \\
& \quad+\int_{0}^{t}\left(\|(m, \boldsymbol{u}, y, \varphi)(\cdot, t)\|_{H^{3}\left(\mathbb{R}^{d}\right)}^{2}+\left\|\left(m_{t}, \boldsymbol{u}_{t}, y_{t}, \varphi_{t}\right)(\cdot, t)\right\|_{H^{2}\left(\mathbb{R}^{d}\right)}^{2}\right) \mathrm{d} t \rightarrow \infty \quad \text { as } t \rightarrow T_{\max } .
\end{aligned}
$$

In order to prove Theorem 3.1, from the standard continuation arguments, we only need an a priori estimate on $(m, \boldsymbol{u}, y, \varphi)$, which is presented in Lemma 3.3. From it, we can extend the local solution in Lemma 3.2 to global existence. This method is a modification of a method introduced in [23] for the compressible Navier-Stokes equations (we omit the details).

Lemma 3.3. Suppose that $(m, \boldsymbol{u}, y, \varphi)$ satisfies the system (3.1), (3.2) for $(x, t) \in$ $\mathbb{R}^{d} \times\left[0, T_{\max }\right)$. Then there exist some positive constants $\delta_{1}, \alpha$ and $C$ depending only on $b(x)$ and $N(x)$ such that, for any $0<S<T_{\max }$, if

$$
\sup _{0 \leqslant t \leqslant S}\left(\|(m, \boldsymbol{u}, \varphi)\|_{H^{3}\left(\mathbb{R}^{d}\right)}+\|y\|_{H^{2}\left(\mathbb{R}^{d}\right)}+\left\|\left(m_{t}, \boldsymbol{u}_{t}, \varphi_{t}, y_{t}\right)\right\|_{H^{2}\left(\mathbb{R}^{d}\right)}\right)+\|\nabla b(x)\|_{H^{5}\left(\mathbb{R}^{d}\right)} \leqslant \delta_{1},
$$

then the estimate (3.3) holds for any $t \in(0, S]$.

In the following we thus focus on the proof of Lemma 3.3. Due to the friction term, $\boldsymbol{u}$, the diffusion term of $y_{x x}$ and the fact that $N(x)>0$, we can prove Lemma 3.3 by the elementary energy method. Obviously, using Lemma 2.1, the a priori assumption (3.4) and Sobolev's inequality, we have

$$
\begin{aligned}
& \sup _{x \in \mathbb{R}^{d}}\left|\left(m, \partial_{x} m, m_{t}, \boldsymbol{u}, \partial_{x} \boldsymbol{u}, \boldsymbol{u}_{t}, \varphi, \partial_{x} \varphi, \varphi_{t}, y, y_{t}, \partial_{x} N, \partial_{x}^{2} N, \partial_{x}^{3} N, \partial_{x}^{4} N\right)\right| \\
& \leqslant C\left(\|(m, \boldsymbol{u}, \varphi)\|_{H^{3}\left(\mathbb{R}^{d}\right)}+\left\|\left(m_{t}, \boldsymbol{u}_{t}, \varphi_{t}, y, y_{t}\right)\right\|_{H^{2}\left(\mathbb{R}^{d}\right)}+\|\nabla N\|_{H^{5}\left(\mathbb{R}^{d}\right)}\right) \\
& \leqslant C \delta_{1},
\end{aligned}
$$

furthermore, we can assume that $\delta_{1}$ is chosen to be so small that, for some $b_{0}>0$,

$$
\frac{1}{2} b_{0} \leqslant N+m \leqslant 2 b_{0}, \quad \frac{1}{2} T^{(0)} \leqslant T^{(0)}+y \leqslant 2 T^{(0)} .
$$

Now we can rewrite $(3.1)_{3}$ as

$$
\Delta y=\frac{3}{2}(N+m) f(x, t)
$$

where

$$
f(x, t)=y_{t}+\boldsymbol{u} \cdot \nabla y+\frac{2}{3}\left(T^{(0)}+y\right) \operatorname{div} \boldsymbol{u}+y-\frac{1}{3}|\boldsymbol{u}|^{2} .
$$

Then, using (3.5) and the standard $L^{2}$-theory of the elliptic operators, we have that

$$
\left\|\partial_{x}^{3} y\right\|^{2} \leqslant C\left(\|f\|^{2}+\left\|\partial_{x} f\right\|^{2}\right) \leqslant C \delta_{1}^{2}
$$

and

$$
\|y\|_{H^{4}\left(\mathbb{R}^{d}\right)}^{2} \leqslant C \delta_{1}^{2}
$$


which imply, by Sobolev's inequality, that

$$
\left\|\left(\partial_{x} y, \partial_{x}^{2} y\right)\right\|_{L^{\infty}} \leqslant C \delta_{1} .
$$

On the other hand, by $(3.1)_{1}$ and (3.5), it is easy to get

$$
\left\|\partial_{x}^{i} m_{t}\right\| \leqslant C \sum_{k=0}^{i+1}\left(\left\|\partial_{x}^{k} \boldsymbol{u}\right\|+\left\|\partial_{x}^{k} m\right\|\right) .
$$

Moreover, after taking $\partial_{x}^{j}, j=1,2,3$, on both sides of $(3.1)_{4}$, multiplying the resulting equation by $\partial_{x}^{j} \Phi$ and then integrating it over $\mathbb{R}^{d}$, integration by parts gives

$$
\int \partial_{x}^{j} \varphi \partial_{x}^{j}(\varphi+E) \mathrm{d} x=\int \partial_{x}^{j-1} m \partial_{x}^{j}(\varphi+E) \mathrm{d} x
$$

which yields

$$
\left\|\partial_{x}^{j} \varphi\right\| \leqslant C\left\|\partial_{x}^{j-1} m\right\| .
$$

Analogously, we can deduce that

$$
\left\|\partial_{x}^{k} \varphi_{t}\right\| \leqslant C \sum_{i=0}^{k}\left(\left\|\partial_{x}^{k} \boldsymbol{u}\right\|+\left\|\partial_{x}^{k} m\right\|\right), \quad k=0,1,2 .
$$

We will now prove Lemma 3.3 using the following three steps.

First, we take $\partial_{t}^{l}(l=0,1)$ on both sides of $(3.1)_{2}$ to give

$$
\partial_{t}^{l} \boldsymbol{u}_{t}+\partial_{t}^{l}((\boldsymbol{u} \cdot \nabla) \boldsymbol{u})+\partial_{t}^{l}\left(\frac{T^{(0)}+y}{N+m} \nabla m+\frac{N y-T^{(0)} m}{N(N+m)} \nabla N+\nabla y\right)=\partial_{t}^{l} \varphi-\partial_{t}^{l} \boldsymbol{u} .
$$

Then we multiply (3.11) by $N \partial_{t}^{l} \boldsymbol{u}$ with $l=0,1$, and integrate the resulting equality over $\mathbb{R}^{d}$. We then see that

$$
\begin{aligned}
& \frac{1}{2} \frac{\mathrm{d}}{\mathrm{d} t} \int N\left|\partial_{t}^{l} \boldsymbol{u}\right|^{2} \mathrm{~d} x+\int N\left|\partial_{t}^{l} \boldsymbol{u}\right|^{2} \mathrm{~d} x \\
& \quad+\int \partial_{t}^{l}\left(\frac{T^{(0)}+y}{N+m} \nabla m-\frac{N y-T^{(0)} m}{N(N+m)} \nabla N\right) N \partial_{t}^{l} \boldsymbol{u} \mathrm{d} x \\
& \quad+\int \partial_{t}^{l} \nabla y \cdot N \partial_{t}^{l} \boldsymbol{u} \mathrm{d} x+\int \partial_{t}^{l}((\boldsymbol{u} \cdot \nabla) \boldsymbol{u}) N \partial_{t}^{l} \boldsymbol{u} \mathrm{d} x-\int \partial_{t}^{l} \varphi N \partial_{t}^{l} \boldsymbol{u} \mathrm{d} x=0
\end{aligned}
$$

We will estimate the integrals in (3.12). First, using integration by parts and (1.11), it follows that

$$
\begin{aligned}
\int\left(\frac{T^{(0)}+y}{N+m} \nabla m \cdot N \boldsymbol{u}+\frac{N y-T^{(0)} m}{N(N+m)} \nabla N \cdot N \boldsymbol{u}\right) \mathrm{d} x \\
=-\int \frac{N\left(T^{(0)}+y\right)}{N+m} m \operatorname{div} \boldsymbol{u} \mathrm{d} x+\int \frac{\left(N y-T^{(0)} m\right) \nabla N}{N(N+m)} \cdot N \boldsymbol{u} \mathrm{d} x \\
\quad-\int m \frac{(N+m) \nabla y-\left(T^{(0)}+y\right) \nabla(N+m)}{(N+m)^{2}} \cdot N \boldsymbol{u} \mathrm{d} x-\int m \frac{T^{(0)}+y}{N+m} \nabla N \cdot \boldsymbol{u} \mathrm{d} x
\end{aligned}
$$




$$
\begin{aligned}
& \geqslant \int \frac{N\left(T^{(0)}+y\right)}{N+m} m \frac{m_{t}+\nabla(N+m) \cdot u}{N+m} \mathrm{~d} x-C \delta_{1}\|(m, \nabla m, \boldsymbol{u}, y)\|^{2} \\
& \geqslant \frac{1}{2} \frac{\mathrm{d}}{\mathrm{d} t} \int \frac{N\left(T^{(0)}+y\right)}{(N+m)^{2}} m^{2} \mathrm{~d} x-C \delta_{1}\|(m, \nabla m, \boldsymbol{u}, y)\|^{2},
\end{aligned}
$$

with the help of (3.5) and (3.7).

Note that in (3.13) we apply the formulation of $\operatorname{div} \boldsymbol{u}$, i.e.

$$
\operatorname{div} \boldsymbol{u}=-\frac{m_{t}+\nabla(N+m) \cdot \boldsymbol{u}}{N+m},
$$

to estimate the nonlinear pressure term. Moreover,

$$
\operatorname{div} \boldsymbol{u}=-\frac{m_{t}+\operatorname{div}(m \boldsymbol{u}) \nabla N \cdot \boldsymbol{u}}{N}
$$

will also be employed to deal with the electric field term below. The two relations above will be repeatedly used in the subsequent analysis, and their advantages will be reflected in establishing higher-order energy estimates. Similarly, we have

$$
\begin{aligned}
& \int \partial_{t}\left(\frac{T^{(0)}+y}{N+m} \nabla m+\frac{N y-T^{(0)} m}{N(N+m)} \nabla N\right) \cdot N \partial_{t} \boldsymbol{u} \mathrm{d} x \\
&=-\int \frac{T^{(0)}+y}{N+m} m_{t} \operatorname{div}\left(N \partial_{t} \boldsymbol{u}\right) \mathrm{d} x \\
& \quad-\int m_{t} \frac{(N+m) \nabla y-\left(T^{(0)}+y\right) \nabla(N+m)}{(N+m)^{2}} \cdot N \partial_{t} \boldsymbol{u} \mathrm{d} x \\
& \quad+\int \partial_{t}\left(\frac{T^{(0)}+y}{N+m}\right) \nabla m \cdot N \partial_{t} \boldsymbol{u} \mathrm{d} x+\int \partial_{t}\left(\frac{N y-T^{(0)} m}{N(N+m)}\right) \nabla N \cdot N \partial_{t} \boldsymbol{u} \mathrm{d} x \\
& \geqslant \int \frac{N\left(T^{(0)}+y\right)}{N+m} m_{t} \partial_{t}\left(\frac{m_{t}+\nabla(N+m) \cdot \boldsymbol{u}}{N+m}\right) \mathrm{d} x-C \delta_{1}\left\|\left(m_{t}, \nabla m, \boldsymbol{u}_{t}, y_{t}\right)\right\|^{2} \\
& \geqslant \frac{1}{2} \frac{\mathrm{d}}{\mathrm{d} t} \int \frac{N\left(T^{(0)}+y\right)}{(N+m)^{2}} m_{t}^{2} \mathrm{~d} x-C \delta_{1}\left\|\left(m_{t}, \nabla m, \boldsymbol{u}_{t}, y_{t}\right)\right\|^{2} .
\end{aligned}
$$

On the other hand, direct computation and (1.11), (3.5) and (3.7) lead to

$$
\int \partial_{t}^{l}((\boldsymbol{u} \cdot \nabla) \boldsymbol{u}) N \partial_{t}^{l} \boldsymbol{u} \mathrm{d} x \geqslant-C \delta_{1} \int\left(\left|\partial_{t}^{l} \boldsymbol{u}\right|^{2}+|\nabla \boldsymbol{u}|^{2}\right) \mathrm{d} x, \quad l=0,1,
$$

and

$$
\begin{aligned}
\int N\left(\nabla y \cdot \boldsymbol{u}+\nabla y_{t} \cdot \partial_{t} \boldsymbol{u}\right) \mathrm{d} x & =-\int\left(y \nabla N \cdot \boldsymbol{u}+N y \operatorname{div} \boldsymbol{u}+y_{t} \nabla N \cdot \boldsymbol{u}_{t}+N y_{t} \operatorname{div} \boldsymbol{u}_{t}\right) \mathrm{d} x \\
& \geqslant-\int N\left(y \operatorname{div} u+y_{t} \operatorname{div} \boldsymbol{u}_{t}\right) \mathrm{d} x-C \delta_{1}\left\|\left(\boldsymbol{u}, \boldsymbol{u}_{t}, y, y_{t}\right)\right\|^{2}
\end{aligned}
$$


By $(3.1)_{1},(3.1)_{4}$ and (1.11), it can be shown that

$$
\begin{aligned}
-\int \varphi \cdot N \boldsymbol{u} \mathrm{d} x & =-\int \Phi\left(\operatorname{div} \varphi_{t}+\operatorname{div}(m \boldsymbol{u})\right) \mathrm{d} x+\int E N \boldsymbol{u} \mathrm{d} x \\
& =\int \varphi\left(\varphi_{t}+m \boldsymbol{u}\right) \mathrm{d} x+\int E\left(\varphi_{t}+(m+N) \boldsymbol{u}\right) \mathrm{d} x \\
& \geqslant \frac{1}{2} \frac{\mathrm{d}}{\mathrm{d} t} \int|\varphi|^{2} \mathrm{~d} x-C \delta_{1}\|(\boldsymbol{u}, \varphi)\|^{2}
\end{aligned}
$$

and

$$
\begin{aligned}
-\int \varphi_{t} \cdot \partial_{t}(N \boldsymbol{u}) \mathrm{d} x & =-\int \partial_{t} \Phi \partial_{t}\left(\operatorname{div} \varphi_{t}+\operatorname{div} m \boldsymbol{u}\right) \mathrm{d} x \\
& =\int \partial_{t} \varphi\left(\partial_{t} \varphi_{t}+\partial_{t}(m \boldsymbol{u})\right) \mathrm{d} x \\
& \geqslant \frac{1}{2} \frac{\mathrm{d}}{\mathrm{d} t} \int\left|\varphi_{t}\right|^{2} \mathrm{~d} x-C \delta_{1}\left\|\left(\boldsymbol{u}_{t}, \varphi_{t}\right)\right\|^{2}
\end{aligned}
$$

where we have used the fact that

$$
\int \partial_{x}^{i} E \partial_{x}^{i}\left(\varphi_{t}+(N+m) \boldsymbol{u}\right)=0, \quad i=0,1,2,3
$$

Indeed, from $(3.1)_{1}$ and $(3.1)_{4}$, we get

$$
\partial_{x}^{i} \operatorname{div}\left(\varphi_{t}+(N+m) \boldsymbol{u}\right)=0 .
$$

Multiplying the above equality by $\partial_{x}^{i-1}\left(T^{(0)} \nabla N / N\right)$ and integrating the resultant equality in $\mathbb{R}^{d}$, we immediately obtain (3.19), with the help of integration by parts and the relation

$$
\partial_{x}^{i}\left(\frac{T^{(0)} \nabla N}{N}\right)=\partial_{x}^{i} E .
$$

Thus, (3.12), together with (3.13)-(3.18), implies that

$$
\begin{gathered}
\frac{1}{2} \frac{\mathrm{d}}{\mathrm{d} t} \int\left(N\left(|\boldsymbol{u}|^{2}+\left|\boldsymbol{u}_{t}\right|^{2}\right)+\frac{N\left(T^{(0)}+y\right)}{(N+m)^{2}}\left(m^{2}+m_{t}^{2}\right)+|\varphi|^{2}+\left|\varphi_{t}\right|^{2}\right) \mathrm{d} x+C\left\|\left(\boldsymbol{u}, \boldsymbol{u}_{t}\right)\right\|^{2} \\
\leqslant C \delta_{1}\left\|\left(m, m_{t}, \nabla m, \nabla \boldsymbol{u}, \varphi, \varphi_{t}, y, y_{t}\right)\right\|^{2}+\int N\left(y \operatorname{div} \boldsymbol{u}+y_{t} \operatorname{div} \boldsymbol{u}_{t}\right) \mathrm{d} x
\end{gathered}
$$

Let us take $\partial_{t}^{l}(l=0,1)$ on both sides of $(3.1)_{3}$, multiply the resulting equation by $N \partial_{t}^{l} y$, and integrate it over $\mathbb{R}^{d}$. Then we obtain

$$
\begin{aligned}
& \frac{1}{2} \frac{\mathrm{d}}{\mathrm{d} t} \int N\left|\partial_{t}^{l} y\right|^{2} \mathrm{~d} x+\int N\left|\partial_{t}^{l} y\right|^{2} \mathrm{~d} x \\
& \quad+\frac{2}{3} T^{(0)} \int N \partial_{t}^{l} y \partial_{t}^{l} \operatorname{div} \boldsymbol{u} \mathrm{d} x-\frac{2}{3} \int \partial_{t}^{l}\left(\frac{\Delta y}{N+m}\right) N \partial_{t}^{l} y \mathrm{~d} x \\
& \quad+\int \partial_{t}^{l}\left(\boldsymbol{u} \cdot \nabla y+\frac{2}{3} y \operatorname{div} \boldsymbol{u}-\frac{1}{3}|\boldsymbol{u}|^{2}\right) N \partial_{t}^{l} y \mathrm{~d} x=0, \quad l=0,1 .
\end{aligned}
$$


It is easy to verify that

$$
\int \partial_{t}^{l}\left(\boldsymbol{u} \cdot \nabla y+\frac{2}{3} y \operatorname{div} \boldsymbol{u}-\frac{1}{3}|\boldsymbol{u}|^{2}\right) N \partial_{t}^{l} y \mathrm{~d} x \geqslant-C \delta_{1}\left\|\left(\boldsymbol{u}, \boldsymbol{u}_{t}, y, y_{t}, \nabla y, \nabla y_{t}\right)\right\|^{2} .
$$

For the fourth integral on the left-hand side of (3.21), using (3.5), (3.7) and (1.11), we can approximate as follows:

$$
\begin{aligned}
-\frac{2}{3} \int \frac{1}{N+m} \Delta y N y \mathrm{~d} x & =\frac{2}{3} \int \nabla y \cdot \frac{(N+m) \nabla(N y)-N y \nabla(N+m)}{(N+m)^{2}} \mathrm{~d} x \\
& \geqslant \int \frac{2 N}{3(N+m)}|\nabla y|^{2} \mathrm{~d} x-C \delta_{1}\|(y, \nabla y)\|^{2}
\end{aligned}
$$

and

$$
\begin{aligned}
-\frac{2}{3} \int \partial_{t}\left(\frac{1}{N+m} \Delta y\right) N \partial_{t} y \mathrm{~d} x & =-\frac{2}{3} \int \frac{(N+m) \Delta y_{t}-\Delta y m_{t}}{(N+m)^{2}} N \partial_{t} y \mathrm{~d} x \\
& \geqslant \int \frac{2 N}{3(N+m)}\left|\nabla y_{t}\right|^{2} \mathrm{~d} x-C \delta_{1}\left\|\left(m_{t}, y_{t}, \nabla y_{t}\right)\right\|^{2} .
\end{aligned}
$$

Inequalities (3.21)-(3.24) show that

$$
\begin{aligned}
\frac{1}{2} \frac{\mathrm{d}}{\mathrm{d} t} \int N\left(y^{2}+\right. & \left.y_{t}^{2}\right) \mathrm{d} x+C\left\|\left(y, y_{t}, \nabla y, \nabla y_{t}\right)\right\|^{2} \\
& +\frac{2}{3} T^{(0)} \int N\left(y \operatorname{div} \boldsymbol{u}+y_{t} \operatorname{div} \boldsymbol{u}_{t}\right) \mathrm{d} x \leqslant C \delta_{1}\left\|\left(m_{t}, \boldsymbol{u}, \boldsymbol{u}_{t}\right)\right\|^{2} .
\end{aligned}
$$

Combining (3.20) and (3.25), we discover that

$$
\begin{aligned}
& \frac{1}{2} \frac{\mathrm{d}}{\mathrm{d} t} \int\left(N\left(|\boldsymbol{u}|^{2}+\left|\boldsymbol{u}_{t}\right|^{2}\right)+\right. \frac{N\left(T^{(0)}+y\right)}{(N+m)^{2}}\left(m^{2}+m_{t}^{2}\right) \\
&\left.+|\varphi|^{2}+\left|\varphi_{t}\right|^{2}+\frac{3 N}{2 T^{(0)}}\left(y^{2}+y_{t}^{2}\right)\right) \mathrm{d} x \\
&+C\left\|\left(\boldsymbol{u}, \boldsymbol{u}_{t}, y, y_{t}, \nabla y, \nabla y_{t}\right)\right\|^{2} \leqslant C \delta_{1}\left\|\left(m, m_{t}, \nabla m, \nabla \boldsymbol{u}\right)\right\|^{2} .
\end{aligned}
$$

We take div on both sides of $(3.1)_{2}$, multiply the resulting equation by $\operatorname{div} \boldsymbol{u}$ and then integrate it over $\mathbb{R}^{d}$. Integration by parts leads to

$$
\begin{array}{r}
\frac{1}{2} \frac{\mathrm{d}}{\mathrm{d} t}\|\operatorname{div} \boldsymbol{u}\|^{2}+\|\operatorname{div} \boldsymbol{u}\|^{2}=-\int \operatorname{div}\left(\frac{T^{(0)}+y}{N+m} \nabla m+\nabla y+\frac{N y-T^{(0)} m}{N(N+m)} \nabla N\right) \operatorname{div} \boldsymbol{u} \mathrm{d} x \\
+\int \operatorname{div} \varphi \operatorname{div} \boldsymbol{u} \mathrm{d} x-\int \operatorname{div}((\boldsymbol{u} \cdot \nabla) \boldsymbol{u}) \operatorname{div} \boldsymbol{u} \mathrm{d} x .
\end{array}
$$


Using $(3.1)_{1},(1.11),(3.5),(3.7)$ and integration by parts, we have

$$
\begin{aligned}
- & \int \operatorname{div}\left(\frac{T^{(0)}+y}{N+m} \nabla m+\nabla y+\frac{N y-T^{(0)} m}{N(N+m)} \nabla N\right) \operatorname{div} \boldsymbol{u} \mathrm{d} x \\
= & \int \frac{T^{(0)}+y}{N+m} \nabla m \nabla \operatorname{div} \boldsymbol{u} \mathrm{d} x-\int \Delta y \operatorname{div} \boldsymbol{u} \mathrm{d} x-\int \operatorname{div}\left(\frac{N y-T^{(0)} m}{N(N+m)} \nabla N\right) \operatorname{div} \boldsymbol{u} \mathrm{d} x \\
=- & \int \frac{T^{(0)}+y}{N+m} \nabla m \nabla\left(\frac{m_{t}+\nabla(N+m) \cdot \boldsymbol{u}}{N+m}\right) \mathrm{d} x-\int \operatorname{div}\left(\frac{N y-T^{(0)} m}{N(N+m)} \nabla N\right) \operatorname{div} \boldsymbol{u} \mathrm{d} x \\
& -\int \frac{3(N+m)}{2}\left(y_{t}+\boldsymbol{u} \cdot \nabla y+\frac{2}{3} y \operatorname{div} \boldsymbol{u}+y-\frac{1}{3}|\boldsymbol{u}|^{2}+\frac{2}{3} T^{(0)} \operatorname{div} \boldsymbol{u}\right) \operatorname{div} \boldsymbol{u} \mathrm{d} x \\
\leqslant- & \frac{1}{2} \int \frac{T^{(0)}+y}{(N+m)^{2}}|\nabla m|^{2} \mathrm{~d} x-T^{(0)} \int(N+m)|\operatorname{div} \boldsymbol{u}|^{2} \mathrm{~d} x \\
\quad & +\varepsilon\|\nabla \boldsymbol{u}\|^{2}+C \delta_{1}\|(m, \nabla m, \boldsymbol{u}, \nabla \boldsymbol{u}, y, \nabla y)\|^{2}+C(\varepsilon)\left\|\left(y, y_{t}\right)\right\|^{2},
\end{aligned}
$$

where $\varepsilon$ is a small positive number; it will appear repeatedly below. Analogously to (3.15) and (3.17), we have

$$
\begin{aligned}
-\int \operatorname{div}((\boldsymbol{u} \cdot \nabla) \boldsymbol{u}) \operatorname{div} \boldsymbol{u} \mathrm{d} x & =-\int \partial_{i}\left(\boldsymbol{u}^{j} \partial_{j} \boldsymbol{u}^{i}\right) \partial_{k} \boldsymbol{u}^{k} \mathrm{~d} x \\
& =-\int \partial_{i} \boldsymbol{u}^{j} \partial_{j} \boldsymbol{u}^{i} \operatorname{div} \boldsymbol{u} \mathrm{d} x+\int \frac{1}{2}(\operatorname{div} \boldsymbol{u})^{3} \mathrm{~d} x \\
& \leqslant C \delta_{1} \int|\nabla \boldsymbol{u}|^{2}
\end{aligned}
$$

and

$$
\begin{aligned}
\int \operatorname{div} \varphi \operatorname{div} \boldsymbol{u} \mathrm{d} x & =-\int m\left(\frac{m_{t}+\operatorname{div}(m \boldsymbol{u}) \mathrm{d} x+\nabla N \cdot \boldsymbol{u}}{N}\right) \\
& \leqslant-\frac{1}{2} \frac{\mathrm{d}}{\mathrm{d} t} \int \frac{1}{N} m^{2} \mathrm{~d} x+C \delta_{1}\|(m, \nabla m, \boldsymbol{u})\|^{2} .
\end{aligned}
$$

Therefore, (3.27)-(3.30) lead to

$$
\begin{aligned}
& \frac{1}{2} \frac{\mathrm{d}}{\mathrm{d} t}\left\|\left(\operatorname{div} \boldsymbol{u}, N^{-1 / 2} m,\left(\frac{T^{(0)}+y}{(N+m)^{2}}\right)^{1 / 2} \nabla m\right)\right\|^{2}+C\|\operatorname{div} \boldsymbol{u}\|^{2} \\
& \leqslant C \delta_{1}\|(m, \nabla m, \boldsymbol{u}, \nabla \boldsymbol{u}, y, \nabla y)\|^{2}+\varepsilon\|\nabla \boldsymbol{u}\|^{2}+C(\varepsilon)\left\|\left(y, y_{t}\right)\right\|^{2} .
\end{aligned}
$$

Analogous to (3.27), we take curl on the two sides of $(3.1)_{2}$ and multiply the resulting equation by $\operatorname{curl} \boldsymbol{u}$ in $L^{2}\left(\mathbb{R}^{d}\right)$ to get

$$
\begin{aligned}
\frac{1}{2} \frac{\mathrm{d}}{\mathrm{d} t}\|\operatorname{curl} \boldsymbol{u}\|^{2}+\|\operatorname{curl} \boldsymbol{u}\|^{2}= & -\int \operatorname{curl}\left(\frac{T^{(0)}+y}{N+m} \nabla m+\frac{N y-T^{(0)} m}{N(N+m)} \nabla N\right) \operatorname{curl} \boldsymbol{u} \mathrm{d} x \\
& -\int \operatorname{curl}((\boldsymbol{u} \cdot \nabla) \boldsymbol{u}) \operatorname{curl} \boldsymbol{u} \mathrm{d} x
\end{aligned}
$$


Direct computation gives

$$
\begin{aligned}
-\int \operatorname{curl}( & \left.\frac{T^{(0)}+y}{N+m} \nabla m\right) \operatorname{curl} \boldsymbol{u} \mathrm{d} x-\int \operatorname{curl}\left(\frac{N y-T^{(0)} m}{N(N+m)} \nabla N\right) \operatorname{curl} \boldsymbol{u} \mathrm{d} x \\
\quad & -\frac{1}{2} \int\left(\partial_{k}\left(\frac{T^{(0)}+y}{N+m}\right) \partial_{i} m-\partial_{i}\left(\frac{T^{(0)}+y}{N+m}\right) \partial_{k} m\right)\left(\partial_{k} \boldsymbol{u}^{i}-\partial_{i} \boldsymbol{u}^{k}\right) \mathrm{d} x \\
& -\frac{1}{2} \int\left(\partial_{k}\left(\frac{N y-T^{(0)} m}{N(N+m)}\right) \partial_{i} N-\partial_{i}\left(\frac{N y-T^{(0)} m}{N(N+m)}\right) \partial_{k} N\right)\left(\partial_{k} \boldsymbol{u}^{i}-\partial_{i} \boldsymbol{u}^{k}\right) \mathrm{d} x \\
& \leqslant C \delta_{1}\|(m, \nabla m, \nabla \boldsymbol{u}, y, \nabla y)\|^{2}
\end{aligned}
$$

and

$$
\begin{aligned}
& -\int \operatorname{curl}((\boldsymbol{u} \cdot \nabla) \boldsymbol{u}) \operatorname{curl} \boldsymbol{u} \mathrm{d} x \\
& \quad=-\frac{1}{2} \int\left(\partial_{k}\left(\boldsymbol{u}^{j} \partial_{j} \boldsymbol{u}^{i}\right)-\partial_{i}\left(\boldsymbol{u}^{j} \partial_{j} \boldsymbol{u}^{k}\right)\right)\left(\partial_{k} \boldsymbol{u}^{i}-\partial_{i} \boldsymbol{u}^{k}\right) \mathrm{d} x \\
& \quad=-\frac{1}{2} \int\left(\partial_{k} \boldsymbol{u}^{j} \partial_{j} \boldsymbol{u}^{i}-\partial_{i} \boldsymbol{u}^{j} \partial_{j} \boldsymbol{u}^{k}\right)\left(\partial_{k} \boldsymbol{u}^{i}-\partial_{i} \boldsymbol{u}^{k}\right) \mathrm{d} x-\frac{1}{2} \int \boldsymbol{u}^{j} \partial_{j}\left(\frac{(\operatorname{curl} \boldsymbol{u})^{2}}{2}\right) \mathrm{d} x \\
& \quad \leqslant C \delta_{1}\|\nabla \boldsymbol{u}\|^{2} .
\end{aligned}
$$

Therefore, Equation (3.32), together with (3.33) and (3.34), implies that

$$
\frac{1}{2} \frac{\mathrm{d}}{\mathrm{d} t}\|\operatorname{curl} \boldsymbol{u}\|^{2}+\|\operatorname{curl} \boldsymbol{u}\|^{2} \leqslant C \delta_{1}\|(y, m, \nabla m, \nabla \boldsymbol{u}, \nabla y)\|^{2} .
$$

Combining (3.31) and (3.35), we have

$$
\begin{aligned}
\frac{1}{2} \frac{\mathrm{d}}{\mathrm{d} t} \int\left(|\nabla \boldsymbol{u}|^{2}+\frac{1}{N} m^{2}+\frac{T^{(0)}+y}{(N+m)^{2}}|\nabla m|^{2}\right) \mathrm{d} x+C|\nabla \boldsymbol{u}|^{2} & \\
& \leqslant C \delta_{1}\|(m, \nabla m, \boldsymbol{u}, y, \nabla y)\|^{2}+C(\varepsilon)\left\|\left(y, y_{t}\right)\right\|^{2}
\end{aligned}
$$

On the other hand, we multiply $(3.1)_{2}$ by $\nabla m$ in $L^{2}\left(\mathbb{R}^{d}\right)$ to get

$$
\begin{aligned}
\int \frac{T^{(0)}+y}{N+m}|\nabla m|^{2} \mathrm{~d} x & \\
& =\int\left(\varphi-\left(\boldsymbol{u}_{t}+\boldsymbol{u}+\nabla y+(\boldsymbol{u} \cdot \nabla) \boldsymbol{u}+\frac{N y-T^{(0)} m}{N(N+m)} \nabla N\right)\right) \cdot \nabla m \mathrm{~d} x \\
& \leqslant-\|m\|^{2}+C\left\|\left(\boldsymbol{u}, \boldsymbol{u}_{t}, \nabla y\right)\right\|^{2}+\varepsilon\|\nabla m\|^{2}+C \delta_{1}\|(m, \nabla m, \nabla \boldsymbol{u}, y)\|^{2}
\end{aligned}
$$

which yields

$$
\|(m, \nabla m)\|^{2} \leqslant C\left\|\left(\boldsymbol{u}, \boldsymbol{u}_{t}, \nabla y\right)\right\|^{2}+C \delta_{1}\|(\nabla \boldsymbol{u}, y)\|^{2} .
$$


Combining (3.26), (3.36), (3.37) and (3.8) with $i=0$ and (3.10) with $k=0$, we obtain

$$
\begin{aligned}
& \frac{\mathrm{d}}{\mathrm{d} t} \int\left(|\boldsymbol{u}|^{2}+\left|\boldsymbol{u}_{t}\right|^{2}+\frac{T^{(0)}+y}{(N+m)^{2}}\left(m^{2}+m_{t}^{2}\right)+\frac{1}{N}\left(|\varphi|^{2}+\left|\varphi_{t}\right|^{2}\right)\right. \\
& \left.+\eta_{1}\left(|\nabla \boldsymbol{u}|^{2}+\frac{1}{N} m^{2}+\frac{T^{(0)}+y}{(N+m)^{2}}|\nabla m|^{2}\right)+\frac{3}{2 T^{(0)}}\left(y^{2}+y_{t}^{2}\right)\right) \mathrm{d} x \\
& +C\left\|\left(m, m_{t}, \nabla m, \boldsymbol{u}, \boldsymbol{u}_{t}, \nabla \boldsymbol{u}, \varphi_{t}, y, y_{t}, \nabla y, \nabla y_{t}\right)\right\|^{2} \leqslant 0
\end{aligned}
$$

for some proper positive constants $\eta_{1}$, depending only on $N(x)$.

By $(3.1)_{2}$, we have

$$
\varphi=\boldsymbol{u}_{t}+(\boldsymbol{u} \cdot \nabla) \boldsymbol{u}+\frac{T^{(0)}+y}{N+m} \nabla m+\frac{N y-T^{(0)} m}{N(N+m)} \nabla N+\nabla y+\boldsymbol{u},
$$

which implies that

$$
\|\varphi\|^{2} \leqslant C\left\|\left(m, \nabla m, m_{t}, \boldsymbol{u}, \boldsymbol{u}_{t}, \nabla \boldsymbol{u}, y, \nabla y\right)\right\|^{2} .
$$

Analogous to (3.18), we have

$$
\begin{aligned}
-\int \partial_{x} \varphi \partial_{x}(N \boldsymbol{u}) \mathrm{d} x=-\int \partial_{x} \Phi \partial_{x} & \operatorname{div} \varphi_{t} \mathrm{~d} x \\
& -\int \partial_{x} \Phi \partial_{x} \operatorname{div}(m \boldsymbol{u}) \mathrm{d} x+\int \partial_{x} E \partial_{x}(m \boldsymbol{u}+N \boldsymbol{u}) \mathrm{d} x
\end{aligned}
$$

which implies

$$
\frac{1}{2} \frac{\mathrm{d}}{\mathrm{d} t} \int\left|\varphi_{x}\right|^{2} \mathrm{~d} x \leqslant C\|(\nabla m, \nabla \boldsymbol{u}, \nabla \varphi)\|^{2} .
$$

Hence, (3.38), together with (3.39), (3.40) and (3.9) with $j=1$, leads to

$$
\begin{aligned}
\frac{\mathrm{d}}{\mathrm{d} t} \int\left(|\boldsymbol{u}|^{2}+\left|\boldsymbol{u}_{t}\right|^{2}\right. & +\frac{T^{(0)}+y}{(N+m)^{2}}\left(m^{2}+m_{t}^{2}\right)+\frac{1}{N}\left(|\varphi|^{2}+\left|\varphi_{t}\right|^{2}\right)+\lambda_{1}|\nabla \varphi|^{2} \\
+\eta_{1}\left(|\nabla \boldsymbol{u}|^{2}\right. & \left.\left.+\frac{1}{N} m^{2}+\frac{T^{(0)}+y}{(N+m)^{2}}|\nabla m|^{2}\right)+\frac{3}{2 T^{(0)}}\left(|y|^{2}+\left|y_{t}\right|^{2}\right)\right) \mathrm{d} x \\
& +C\left\|\left(m, m_{t}, \nabla m, \boldsymbol{u}, \boldsymbol{u}_{t}, \nabla \boldsymbol{u}, \varphi, \varphi_{t}, \nabla \varphi, y, y_{t}, \nabla y, \nabla y_{t}\right)\right\|^{2} \leqslant 0
\end{aligned}
$$

for some appropriate positive constant $\lambda_{1}$, only depends on $N(x)$.

Next, we establish the estimates for the second-order derivatives. Applying a method similar to that for $(3.36),(3.37)$ and (3.38) to

$$
\int \partial_{x}^{2}(3.1)_{2} \partial_{x}^{2}(N \boldsymbol{u}) \mathrm{d} x, \quad \int \partial_{x} \partial_{t}(3.1)_{2} \partial_{x} \partial_{t}(N \boldsymbol{u}) \mathrm{d} x \quad \text { and } \quad \int \partial_{x}(3.1)_{2} \partial_{x} \nabla m \mathrm{~d} x
$$


respectively, by using (3.5), (3.7) and (1.11). Thus, we have

$$
\begin{aligned}
& \frac{\mathrm{d}}{\mathrm{d} t} \int\left(\left|\partial_{x}^{2} \boldsymbol{u}\right|^{2} \mathrm{~d} x+\frac{2}{N}\left(\partial_{i} N \partial_{x}^{2} \boldsymbol{u} \partial_{j} \boldsymbol{u}+\cdots+\boldsymbol{u} \partial_{x}^{2} N \partial_{x}^{2} \boldsymbol{u}\right)\right. \\
& \left.+\frac{T^{(0)}+y}{(N+m)^{2}}\left|\partial_{x}^{2} m\right|^{2}+\frac{1}{N}\left|\partial_{x}^{2} \varphi\right|^{2}\right) \mathrm{d} x \\
& +C \int N(N+m)|\nabla \operatorname{div} \boldsymbol{u}|^{2} \mathrm{~d} x+C\left\|\partial_{x}^{2} \boldsymbol{u}\right\|^{2} \\
& \leqslant C(\varepsilon)\left\|\left(\nabla y, \nabla y_{t}\right)\right\|^{2}+C \delta_{1}\left\|\left(m_{t}, \partial_{x} m_{t}, \boldsymbol{u}_{t}, \partial_{x} \boldsymbol{u}_{t}, y_{t}, \partial_{x} y_{t}, \partial_{x}^{2} \varphi\right)\right\|^{2} \\
& +C \delta_{1}\left(\|(m, y)\|_{H^{2}\left(\mathbb{R}^{d}\right)}^{2}+\|\boldsymbol{u}\|_{H^{1}\left(\mathbb{R}^{d}\right)}^{2}\right), \\
& \frac{1}{2} \frac{\mathrm{d}}{\mathrm{d} t} \int\left(N\left|\partial_{x} \boldsymbol{u}_{t}\right|^{2}+2 \partial_{x} N \partial_{x} \boldsymbol{u}_{t} \boldsymbol{u}_{t}+\frac{N\left(T^{(0)}+y\right)}{(N+m)^{2}}\left|\partial_{x} m_{t}\right|^{2}+\left|\partial_{x} \varphi_{t}\right|^{2}\right) \mathrm{d} x \\
& \leqslant C\|(m, \boldsymbol{u})\|_{H^{1}\left(\mathbb{R}^{d}\right)}^{2}+\int N \partial_{x} y_{t} \partial_{x} \operatorname{div} \boldsymbol{u}_{t} \mathrm{~d} x \\
& +C \delta_{1}\left\|\left(m_{t}, \partial_{x} m_{t}, \partial_{x}^{2} m, \boldsymbol{u}_{t}, \partial_{x}^{2} \boldsymbol{u}, \varphi_{t}, y_{t}, y_{x}, \nabla y_{t}\right)\right\|^{2}, \\
& \left\|\left(\partial_{x} m, \partial_{x}^{2} m\right)\right\|^{2} \leqslant C\left\|\left(\partial_{x} \boldsymbol{u}, \partial_{x} \boldsymbol{u}_{t}, \partial_{x}^{2} y\right)\right\|^{2}+C \delta_{1}\left\|\left(m, \boldsymbol{u}, \partial_{x}^{2} \boldsymbol{u}, y, \partial_{x} y\right)\right\|^{2} .
\end{aligned}
$$

Meanwhile, taking into account

we get

$$
\int\left(\partial_{x}(3.1)_{3} N \partial_{x} y+\partial_{x} \partial_{t}(3.1)_{3} N \partial_{x} \partial_{t} y\right) \mathrm{d} x
$$

$$
\begin{array}{r}
\frac{1}{2} \frac{\mathrm{d}}{\mathrm{d} t} \int N\left(\left|\partial_{x} y\right|^{2}+\left|\partial_{x} y_{t}\right|^{2}\right) \mathrm{d} x+C\left\|\left(\partial_{x} y, \partial_{x}^{2} y, \partial_{x}^{2} y_{t}, \partial_{x} y_{t}\right)\right\|^{2}+\frac{2}{3} T^{(0)} \int N \partial_{x} y_{t} \partial_{x} \operatorname{div} \boldsymbol{u}_{t} \mathrm{~d} x \\
\leqslant C \delta_{1}\left\|\left(\partial_{x} \boldsymbol{u}_{t}, \partial_{x}^{2} u, \partial_{x} m_{t}\right)\right\|^{2}+C(\varepsilon)\left\|\partial_{x} \boldsymbol{u}\right\|^{2}
\end{array}
$$

Therefore, combining (3.42), (3.43)-(3.45) and (3.8) with $i=1$ and (3.9) with $j=2$, we conclude

$$
\begin{gathered}
\frac{\mathrm{d}}{\mathrm{d} t} \int\left[\eta_{2}\left(\left|\partial_{x}^{2} \boldsymbol{u}\right|^{2}+\frac{1}{N}\left|\partial_{x}^{2} \varphi\right|^{2}+\frac{T^{(0)}+y}{(N+m)^{2}}\left|\partial_{x}^{2} m\right|^{2}+\frac{2}{N}\left(\partial_{i} N \partial_{j} \boldsymbol{u} \partial_{x}^{2} \boldsymbol{u}+\cdots+\boldsymbol{u} \partial_{x}^{2} N \partial_{x}^{2} \boldsymbol{u}\right)\right)\right. \\
+\left|\partial_{x} \boldsymbol{u}_{t}\right|^{2}+\frac{2}{N} \partial_{x} N \boldsymbol{u}_{t} \partial_{x} \boldsymbol{u}_{t}+\frac{T^{(0)}+y}{(N+m)^{2}}\left|\partial_{x} m_{t}\right|^{2} \\
\left.+\frac{1}{N}\left|\partial_{x} \varphi_{t}\right|^{2}+\frac{3}{2 T(0)}\left(\left|\partial_{x} y\right|^{2}+\left|\partial_{x} y_{t}\right|^{2}\right)\right] \mathrm{d} x \\
+C\left\|\left(\partial_{x} m_{t}, \partial_{x}^{2} m, \partial_{x} \boldsymbol{u}_{t}, \partial_{x}^{2} \boldsymbol{u}, \partial_{x} \varphi_{t}, \partial_{x}^{2} \varphi, \partial_{x} y, \partial_{x} y_{t}, \partial_{x}^{2} y, \partial_{x}^{2} y_{t}\right)\right\|^{2} \\
\leqslant C\left(\|(m, \boldsymbol{u}, \varphi)\|_{H^{1}\left(\mathbb{R}^{d}\right)}^{2}+\|y\|^{2}\right)+C \delta_{1}\left\|\left(m_{t}, \boldsymbol{u}_{t}, \varphi_{t}, y_{t}\right)\right\|^{2}
\end{gathered}
$$

for some positive constant $\eta_{2}$ depending only on $N(x)$. 
Finally, let us turn to the estimates of the third derivatives. Note that in obtaining the estimates on the first and the second derivatives we have used the smallness of

$$
\left|\left(m, \partial_{x} m, \boldsymbol{u}, \partial_{x} \boldsymbol{u}, \varphi, \partial_{x} \varphi, y, \partial_{x} y, \partial_{x}^{2} y\right)\right| \text { and }\left|\left(m_{t}, \boldsymbol{u}_{t}, y_{t}, \varphi_{t}\right)\right|,
$$

which are guaranteed by (3.5), (3.7) and the smallness of $\delta$. However, the above arguments do not work for the third derivatives because we cannot obtain the smallness of $\left|\left(\partial_{x}^{2} m, \partial_{x}^{2} \boldsymbol{u}, \partial_{x}^{2} \varphi, \partial_{x}^{3} y\right)\right|$ and $\left|\left(\partial_{x} m_{t}, \partial_{x} \boldsymbol{u}_{t}, \partial_{x} \varphi_{t}, \partial_{x} y_{t}\right)\right|$. Hence, to get the estimates for the third derivative, we must apply some technique inequalities (1.13)-(1.14) and give a detailed discussion. Let us differentiate $(3.1)_{2}$ with respective to $x^{3}$, multiply by $\partial_{x}^{3}(N \boldsymbol{u})$ and integrate the resulting equation over $\mathbb{R}^{d}$. We have

$$
\begin{aligned}
\int \partial_{x}^{3} \boldsymbol{u}_{t} \partial_{x}^{3}(N \boldsymbol{u}) \mathrm{d} x+ & \int \partial_{x}^{3} \boldsymbol{u} \partial_{x}^{3}(N \boldsymbol{u}) \mathrm{d} x \\
=- & \int \partial_{x}^{3}\left(\frac{T^{(0)}+y}{N+m} \nabla m+\frac{N y-T^{(0)} m}{N(N+m)} \nabla N+\nabla y\right) \partial_{x}^{3}(N \boldsymbol{u}) \mathrm{d} x \\
& \quad-\int \partial_{x}^{3}((\boldsymbol{u} \cdot \nabla) \boldsymbol{u}) \partial_{x}^{3}(N \boldsymbol{u}) \mathrm{d} x+\int \partial_{x}^{3} \varphi \partial_{x}^{3}(N \boldsymbol{u}) \mathrm{d} x \\
=: & I_{1}+I_{2}+I_{3} .
\end{aligned}
$$

We will estimate all integral terms in (3.47). First, a direct computation leads to

$$
\begin{aligned}
\int \partial_{x}^{3} \boldsymbol{u}_{t} \partial_{x}^{3}(N \boldsymbol{u}) \mathrm{d} x+\int \partial_{x}^{3} \boldsymbol{u} \partial_{x}^{3}(N \boldsymbol{u}) \mathrm{d} x \\
=\int\left(N \partial_{x}^{3} \boldsymbol{u}_{t} \partial_{x}^{3} \boldsymbol{u}+\partial_{h} \partial_{i} N \partial_{j} \boldsymbol{u} \partial_{x}^{3} \boldsymbol{u}_{t}+\cdots+\partial_{h} N \partial_{i} \partial_{j} \boldsymbol{u} \partial_{x}^{3} \boldsymbol{u}_{t}+\cdots+u \partial_{x}^{3} N \partial_{x}^{3} \boldsymbol{u}_{t}\right) \mathrm{d} x \\
\quad+\int \partial_{x}^{3} \boldsymbol{u}\left(N \partial_{x}^{3} \boldsymbol{u}+\partial_{h} N \partial_{i} \partial_{j} \boldsymbol{u}+\cdots+\partial_{h} \partial_{i} N \partial_{j} \boldsymbol{u}+\cdots+\boldsymbol{u} \partial_{x}^{3} N\right) \mathrm{d} x \\
\geqslant \frac{\mathrm{d}}{\mathrm{d} t} \int\left(\frac{1}{2} N\left|\partial_{x}^{3} \boldsymbol{u}\right|^{2}+\partial_{h} \partial_{i} N \partial_{j} \boldsymbol{u} \partial_{x}^{3} \boldsymbol{u}+\cdots+\partial_{h} N \partial_{i} \partial_{j} \boldsymbol{u} \partial_{x}^{3} \boldsymbol{u}+\cdots+\boldsymbol{u} \partial_{x}^{3} N \partial_{x}^{3} \boldsymbol{u}\right) \mathrm{d} x \\
\quad+\int N\left|\partial_{x}^{3} \boldsymbol{u}\right|^{2} \mathrm{~d} x-C \delta_{1}\left\|\left(\boldsymbol{u}, \partial_{x} \boldsymbol{u}, \boldsymbol{u}_{t}, \partial_{x} \boldsymbol{u}_{t}, \partial_{x}^{2} \boldsymbol{u}, \partial_{x}^{2} \boldsymbol{u}_{t}, \partial_{x}^{3} \boldsymbol{u}\right)\right\|^{2} .
\end{aligned}
$$

By using $(3.1)_{1},(3.5),(3.7)$ and the Moser-type calculus (1.12), we have

$$
\begin{aligned}
I_{1}=- & \int\left(\partial_{x}^{3}\left(\frac{T^{(0)}+y}{N+m} \nabla m+\frac{N y-T^{(0)} m}{N(N+m)} \nabla N\right)+\partial_{x} \nabla\left(\frac{3}{2}(N+m) f\right)\right) \partial_{x}^{3}(N \boldsymbol{u}) \mathrm{d} x \\
\leqslant- & \int\left(\frac{T^{(0)}+y}{N+m} \partial_{h} \partial_{i} \partial_{j} \nabla m+\partial_{h}\left(\frac{T^{(0)}+y}{N+m}\right) \partial_{i} \partial_{j} \nabla m\right. \\
& \left.\quad+\cdots+\partial_{i} \partial_{j}\left(\frac{T^{(0)}+y}{N+m}\right) \partial_{h} \nabla m+\cdots+\partial_{h} \partial_{i} \partial_{j}\left(\frac{T^{(0)}+y}{N+m}\right) \nabla m\right) \partial_{x}^{3}(N \boldsymbol{u}) \mathrm{d} x \\
& -T^{(0)} \int N(N+m)\left|\partial_{x} \nabla \operatorname{div} \boldsymbol{u}\right|^{2} \mathrm{~d} x+\varepsilon\left\|\left(\partial_{x}^{3} \boldsymbol{u}, \partial_{x}^{2} \boldsymbol{u}\right)\right\|^{2}+C(\varepsilon)\left\|\left(\partial_{x}^{2} y_{t}, \partial_{x}^{2} y\right)\right\|^{2} \\
& +C \delta_{1}\left(\|(m, \boldsymbol{u}, y)\|_{H^{3}\left(\mathbb{R}^{d}\right)}^{2}+\left\|y_{t}\right\|_{H^{2}\left(\mathbb{R}^{d}\right)}^{2}\right)
\end{aligned}
$$




$$
\begin{aligned}
\leqslant- & \int \frac{T^{(0)}+y}{N+m} \partial_{x}^{3} \nabla m \partial_{x}^{3}(N \boldsymbol{u}) \mathrm{d} x-T^{(0)} \int N(N+m)\left|\partial_{x} \nabla \operatorname{div} \boldsymbol{u}\right|^{2} \mathrm{~d} x \\
& +C(\varepsilon)\left\|\left(\partial_{x}^{2} y_{t}, \partial_{x}^{2} y\right)\right\|^{2}+C \delta_{1}\left(\|(m, \boldsymbol{u}, y)\|_{H^{3}\left(\mathbb{R}^{d}\right)}^{2}+\left\|y_{t}\right\|_{H^{2}\left(\mathbb{R}^{d}\right)}^{2}\right) \\
& +C(\varepsilon) \int\left|\partial_{x}^{2} m\right|^{4} \mathrm{~d} x .
\end{aligned}
$$

Let us now turn our attention to the first integral term in (3.49). Using (3.1) 1 , integration by parts, the Cauchy-Schwartz inequality (1.11) and a Moser-type calculus (1.12), we can show that

$$
\begin{aligned}
& -\int \frac{T^{(0)}+y}{N+m} \partial_{x}^{3} \nabla m \partial_{x}^{3}(N \boldsymbol{u}) \mathrm{d} x \\
& =-\int \frac{T^{(0)}+y}{N+m} \partial_{x}^{3} m N \partial_{x}^{3}\left(\frac{m_{t}+\nabla(N+m) \cdot \boldsymbol{u}}{N+m}\right) \mathrm{d} x \\
& +\int\left(\nabla\left(\frac{T^{(0)}+y}{N+m}\right) \partial_{x}^{3} m \partial_{x}^{3}(N \boldsymbol{u})+\frac{T^{(0)}+y}{N+m} \partial_{x}^{3} m \partial_{x}^{3}(\nabla N \boldsymbol{u})\right) \mathrm{d} x \\
& \leqslant \int \frac{N\left(T^{(0)}+y\right)}{N+m} \partial_{x}^{3} m \\
& \times\left(\frac { 1 } { N + m } \left(\partial_{x}^{3} m_{t}+\boldsymbol{u} \cdot \nabla \partial_{h} \partial_{i} \partial_{j}(N+m)+\partial_{h} \boldsymbol{u} \cdot \nabla \partial_{i} \partial_{j}(N+m)\right.\right. \\
& \left.+\cdots+\partial_{h} \partial_{i} \boldsymbol{u} \cdot \nabla \partial_{j}(N+m)+\cdots+\partial_{h} \partial_{i} \partial_{j} \boldsymbol{u} \cdot \nabla(N+m)\right) \\
& -\frac{\partial_{h}(N+m)}{(N+m)^{2}}\left(\partial_{i} \partial_{j} m_{t}+\boldsymbol{u} \cdot \nabla \partial_{i} \partial_{j}(N+m)\right. \\
& \left.+\partial_{i} \boldsymbol{u} \cdot \nabla \partial_{j}(N+m)+\cdots+\partial_{i} \partial_{j} \boldsymbol{u} \cdot \nabla(N+m)\right) \\
& -\cdots-\left(\frac{\partial_{h} \partial_{i}(N+m)}{(N+m)^{2}}-\frac{2 \partial_{h}(N+m) \partial_{i}(N+m)}{(N+m)^{3}}\right) \\
& \times\left(\partial_{j} m_{t}+\boldsymbol{u} \cdot \nabla \partial_{j}(N+m)+\partial_{j} \boldsymbol{u} \cdot \nabla(N+m)\right) \\
& -\cdots-\left(\frac{\partial_{h} \partial_{i} \partial_{j}(N+m)}{(N+m)^{2}}-\frac{2 \partial_{h} \partial_{i}(N+m) \partial_{j}(N+m)+\cdots}{(N+m)^{3}}\right. \\
& \left.+\frac{6 \partial_{h}(N+m) \partial_{i}(N+m) \partial_{j}(N+m)}{(N+m)^{4}}\right) \\
& \left.\times\left(m_{t}+\boldsymbol{u} \cdot \nabla(N+m)\right)\right) \mathrm{d} x \\
& +C \delta_{1}\|(m, \boldsymbol{u})\|_{H^{3}\left(\mathbb{R}^{d}\right)}^{2} \\
& \leqslant-\frac{1}{2} \frac{\mathrm{d}}{\mathrm{d} t} \int \frac{N\left(T^{(0)}+y\right)}{(N+m)^{2}}\left|\partial_{x}^{3} m\right|^{2} \mathrm{~d} x+C \delta_{1}\|(m, \boldsymbol{u})\|_{H^{3}\left(\mathbb{R}^{d}\right)}^{2}+C \delta_{1}\left\|\left(m_{t}, \partial_{x} m_{t}, \partial_{x}^{2} m_{t}\right)\right\|^{2} \\
& +\varepsilon\left\|\partial_{x}^{3} m\right\|^{2}+C(\varepsilon) \int\left(\left|\partial_{x} m_{t}\right|^{4}+\left|\partial_{x}^{2} m\right|^{4}+\left|\partial_{x}^{2} \boldsymbol{u}\right|^{4}\right) \mathrm{d} x .
\end{aligned}
$$


Likewise, we can deduce

$$
\begin{aligned}
I_{2} & =-\int\left(\partial_{h} \partial_{i} \partial_{j} \boldsymbol{u} \nabla \boldsymbol{u}+\partial_{h} \partial_{i} \boldsymbol{u} \partial_{j} \nabla \boldsymbol{u}+\cdots+\partial_{h} \boldsymbol{u} \partial_{i} \partial_{j} \nabla \boldsymbol{u}+\cdots+\boldsymbol{u} \partial_{h} \partial_{i} \partial_{j} \nabla \boldsymbol{u}\right) \partial_{x}^{3}(N \boldsymbol{u}) \mathrm{d} x \\
& \leqslant C \delta_{1}\left\|\left(\boldsymbol{u}, \partial_{x} \boldsymbol{u}, \partial_{x}^{2} \boldsymbol{u}, \partial_{x}^{3} \boldsymbol{u}\right)\right\|^{2}+\varepsilon\left\|\partial_{x}^{3} \boldsymbol{u}\right\|^{2}+C(\varepsilon) \int\left|\partial_{x}^{2} \boldsymbol{u}\right|^{4} \mathrm{~d} x
\end{aligned}
$$

and

$$
\begin{aligned}
I_{3} & =\int \partial_{x}^{3} \varphi \partial_{x}^{3}(N \boldsymbol{u}) \mathrm{d} x \\
& =-\int \partial_{x}^{3} \Phi \partial_{x}^{3} \operatorname{div}(N \boldsymbol{u}) \mathrm{d} x-\int \partial_{x}^{3} E \partial_{x}^{3}(N \boldsymbol{u}) \mathrm{d} x \\
& =-\int \partial_{x}^{3} \varphi\left(\partial_{x}^{3} \varphi_{t}+\partial_{x}^{3}(m \boldsymbol{u})\right) \mathrm{d} x-\int \partial_{x}^{3} E \partial_{x}^{3}\left(\varphi_{t}+(m+N) \boldsymbol{u}\right) \mathrm{d} x \\
& \leqslant-\frac{1}{2} \frac{\mathrm{d}}{\mathrm{d} t} \int\left|\partial_{x}^{3} \varphi\right|^{2} \mathrm{~d} x+C \delta_{1}\left\|\left(\partial_{x}^{2} m, \partial_{x}^{3} m, \partial_{x}^{2} \boldsymbol{u}, \partial_{x}^{3} \boldsymbol{u}, \partial_{x}^{3} \varphi\right)\right\|^{2} .
\end{aligned}
$$

Now it is time to estimate the terms of the form $\int|\cdot|^{4} \mathrm{~d} x$ in the previous relations. Using (3.5), (3.7), (1.11)-(1.14), (3.4) and $d=2,3$ we have

$$
\begin{aligned}
\int\left(\left|\partial_{x}^{2} \boldsymbol{u}\right|^{4}+\left|\partial_{x}^{2} m\right|^{4}+\left|\partial_{x} m_{t}\right|^{4}\right) \mathrm{d} x \\
\quad \leqslant C \int\left(\left|\partial_{x}^{2} \boldsymbol{u}\right|^{4}+\left|\partial_{x}^{2} m\right|^{4}\right) \mathrm{d} x+C \delta_{1}\left\|\left(\partial_{x} m, \partial_{x}^{2} m, \partial_{x} \boldsymbol{u}, \partial_{x}^{2} \boldsymbol{u}\right)\right\|^{2} \\
\leqslant C\left\|\partial_{x}^{2} \boldsymbol{u}\right\|^{4-d}\left\|\partial_{x} \partial_{x}^{2} \boldsymbol{u}\right\|^{d}+C\left\|\partial_{x}^{2} m\right\|^{4-d}\left\|\partial_{x} \partial_{x}^{2} m\right\|^{d}+C \delta_{1}\left\|\left(\partial_{x} m, \partial_{x}^{2} m, \partial_{x} \boldsymbol{u}, \partial_{x}^{2} \boldsymbol{u}\right)\right\|^{2} \\
\quad \leqslant C\left\|\partial_{x}^{2} \boldsymbol{u}\right\|^{4-d}\left\|\partial_{x} \partial_{x}^{2} \boldsymbol{u}\right\|^{d-2}\left\|\partial_{x} \partial_{x}^{2} \boldsymbol{u}\right\|^{2}+C\left\|\partial_{x}^{2} m\right\|^{4-d}\left\|\partial_{x} \partial_{x}^{2} m\right\|^{d-2}\left\|\partial_{x} \partial_{x}^{2} m\right\|^{2} \\
\quad+C \delta_{1}\left\|\left(\partial_{x} m, \partial_{x}^{2} m, \partial_{x} \boldsymbol{u}, \partial_{x}^{2} \boldsymbol{u}\right)\right\|^{2} \\
\quad \leqslant C \delta_{1}\left\|\left(\partial_{x} m, \partial_{x}^{2} m, \partial_{x}^{3} m, \partial_{x} \boldsymbol{u}, \partial_{x}^{2} \boldsymbol{u}, \partial_{x}^{3} \boldsymbol{u}\right)\right\|^{2} .
\end{aligned}
$$

Therefore, (3.47), together with (3.48)-(3.53), implies that

$$
\begin{gathered}
\frac{\mathrm{d}}{\mathrm{d} t} \int\left(\left|\partial_{x}^{3} \boldsymbol{u}\right|^{2} \mathrm{~d} x+\frac{2}{N}\left(\boldsymbol{u} \partial_{x}^{3} N \partial_{x}^{3} \boldsymbol{u}+\partial_{h} N \partial_{x}^{3} \boldsymbol{u} \partial_{i} \partial_{j} \boldsymbol{u}+\cdots+\partial_{h} \partial_{i} N \partial_{x}^{3} \boldsymbol{u} \partial_{j} \boldsymbol{u}+\cdots\right)\right. \\
\left.+\frac{T^{(0)}+y}{(N+m)^{2}}\left|\partial_{x}^{3} m\right|^{2}+\frac{1}{N}\left|\partial_{x}^{3} \varphi\right|^{2}\right) \mathrm{d} x \\
+C \int N(N+m)\left|\partial_{x} \nabla \operatorname{div} \boldsymbol{u}\right|^{2} \mathrm{~d} x+C\left\|\partial_{x}^{3} u\right\|^{2} \\
\leqslant C(\varepsilon)\left\|\left(\partial_{x}^{2} y, \partial_{x}^{2} y_{t}\right)\right\|^{2}+C \delta_{1}\left(\|(m, y)\|_{H^{3}\left(\mathbb{R}^{d}\right)}^{2}+\|\boldsymbol{u}\|_{H^{2}\left(\mathbb{R}^{d}\right)}^{2}\right) \\
+C \delta_{1}\left\|\left(m_{t}, \partial_{x} m_{t}, \partial_{x}^{2} m_{t}, \partial_{x}^{3} \varphi, y_{t}, \partial_{x} y_{t}\right)\right\|^{2} .
\end{gathered}
$$


Analogously to (3.37) and (3.44), we apply the Cauchy-Schwartz inequality (1.11) to the identity

$$
\begin{aligned}
& \int \frac{T^{(0)}+y}{N+m}\left|\partial_{x}^{2} \nabla m\right|^{2} \mathrm{~d} x \\
& =\int \partial_{x}^{2}\left(\varphi-\left(\boldsymbol{u}_{t}+\boldsymbol{u}+\nabla y+(\boldsymbol{u} \cdot \nabla) \boldsymbol{u}+\frac{N y-T^{(0)} m}{N(N+m)} \nabla N\right)\right) \partial_{x}^{2} \nabla m \mathrm{~d} x \\
& \quad-\int \partial_{x}^{2}\left(\frac{T^{(0)}+y}{N+m}\right) \nabla m \partial_{x}^{2} \nabla m \mathrm{~d} x-2 \int \partial_{x}\left(\frac{T^{(0)}+y}{N+m}\right) \partial_{x} \nabla m \partial_{x}^{2} \nabla m \mathrm{~d} x
\end{aligned}
$$

which yields

$$
\begin{aligned}
& \left\|\left(\partial_{x}^{3} m, \partial_{x}^{2} m\right)\right\|^{2} \\
& \quad \leqslant C\left\|\left(\partial_{x}^{2} \boldsymbol{u}, \partial_{x}^{2} \boldsymbol{u}_{t}, \partial_{x}^{3} y\right)\right\|^{2}+C \delta_{1}\left\|\left(m, \partial_{x} m, \partial_{x} \boldsymbol{u}, \partial_{x}^{3} \boldsymbol{u}, y, \partial_{x} y, \partial_{x}^{2} y\right)\right\|^{2} .
\end{aligned}
$$

Next, let us take $\partial_{x}^{2} \partial_{t}$ on both sides of $(3.1)_{2}$, multiply the resulting equation by $\partial_{x}^{2} \partial_{t}(N \boldsymbol{u})$, and integrate it over $\mathbb{R}^{d}$. We discover that

$$
\begin{aligned}
& \int \partial_{x}^{2} \partial_{t} \boldsymbol{u}_{t} \partial_{x}^{2} \partial_{t}(N \boldsymbol{u}) \mathrm{d} x+\int \partial_{x}^{2} \partial_{t} \boldsymbol{u} \partial_{x}^{2} \partial_{t}(N \boldsymbol{u}) \mathrm{d} x \\
&+\int \partial_{x}^{2} \partial_{t}\left(\frac{T^{(0)}+y}{N+m} \nabla m+\frac{N y-T^{(0)} m}{N(N+m)} \nabla N+\nabla y\right) \partial_{x}^{2} \partial_{t}(N \boldsymbol{u}) \mathrm{d} x \\
& \quad+\int \partial_{x}^{2} \partial_{t}((\boldsymbol{u} \cdot \nabla) \boldsymbol{u}) \partial_{x}^{2} \partial_{t}(N \boldsymbol{u}) \mathrm{d} x-\int \partial_{x}^{2} \partial_{t} \varphi \partial_{x}^{2} \partial_{t}(N \boldsymbol{u}) \mathrm{d} x=0
\end{aligned}
$$

In a completely similar way, we can obtain

$$
\begin{aligned}
& \frac{1}{2} \frac{\mathrm{d}}{\mathrm{d} t} \int\left(N\left|\partial_{x}^{2} \boldsymbol{u}_{t}\right|^{2}+2\left(\partial_{i} N \partial_{x}^{2} \boldsymbol{u}_{t} \partial_{j} \boldsymbol{u}_{t}+\cdots\right.\right.\left.+\partial_{x}^{2} N \partial_{x}^{2} \boldsymbol{u}_{t} \boldsymbol{u}_{t}\right) \\
&\left.+\frac{N\left(T^{(0)}+y\right)}{(N+m)^{2}}\left|\partial_{x}^{2} m_{t}\right|^{2}+\left|\partial_{x}^{2} \varphi_{t}\right|^{2}\right) \mathrm{d} x \\
&-\int N \partial_{x}^{2} y_{t} \partial_{x}^{2} \operatorname{div} \boldsymbol{u}_{t} \mathrm{~d} x+C\left\|\left(\partial_{x}^{2} \boldsymbol{u}_{t}, \partial_{x}^{2} \varphi_{t}\right)\right\|^{2} \\
& \leqslant C\|(m, \boldsymbol{u})\|_{H^{2}\left(\mathbb{R}^{d}\right)}^{2}+C \delta_{1}\left(\left\|\left(m_{t}, \boldsymbol{u}_{t}, \varphi_{t}, y_{t}\right)\right\|_{H^{2}\left(\mathbb{R}^{d}\right)}^{2}+\left\|\partial_{x}^{3} m\right\|\right),
\end{aligned}
$$

with the help of (3.10) with $k=2$.

In the following, we define

$$
G_{0}=\frac{1}{2} N\left(\left|\partial_{x}^{2} y\right|^{2}+\left|\partial_{x}^{2} y_{t}\right|^{2}\right)
$$


Deriving $G_{0}$ with respect to $t$, using $(3.1)_{3}$, and integrating it over the whole space, we obtain

$$
\begin{aligned}
\frac{\mathrm{d}}{\mathrm{d} t} \int G_{0} \mathrm{~d} x & +\int N\left(\left|\partial_{x}^{2} y\right|^{2}+\left|\partial_{x}^{2} y_{t}\right|^{2}\right) \mathrm{d} x+\frac{2}{3} T^{(0)} \int N \partial_{x}^{2} y_{t} \partial_{x}^{2} \operatorname{div} \boldsymbol{u}_{t} \mathrm{~d} x \\
=- & -\int\left(\frac{2}{3} T^{(0)} N \partial_{x}^{2} y \partial_{x}^{2} \operatorname{div} \boldsymbol{u}+N \partial_{x}^{2} y \partial_{x}^{2}\left(u \cdot \nabla y+\frac{2}{3} y \operatorname{div} \boldsymbol{u}-\frac{1}{3}|\boldsymbol{u}|^{2}\right)\right. \\
& \left.+N \partial_{x}^{2} y_{t} \partial_{x}^{2} \partial_{t}\left(u \cdot \nabla y-\frac{1}{3}|\boldsymbol{u}|^{2}\right)\right) \mathrm{d} x \\
& \quad-\int \frac{2}{3} N \partial_{x}^{2} y_{t} \partial_{x}^{2} \partial_{t}(y \operatorname{div} \boldsymbol{u}) \mathrm{d} x+\frac{2}{3} \int N\left(\partial_{x}^{2} y \partial_{x}^{2}+\partial_{x}^{2} y_{t} \partial_{x}^{2} \partial_{t}\right) \frac{\Delta y}{N+m} \mathrm{~d} x \\
= & I_{1}+I_{2}+I_{3}+I_{4}
\end{aligned}
$$

Using Moser-type calculus (1.12), it is easy for us to compute

$$
I_{1} \leqslant C\|\boldsymbol{u}\|_{H^{2}\left(\mathbb{R}^{d}\right)}^{2}+C \delta_{1}\|(\boldsymbol{u}, y)\|_{H^{3}\left(\mathbb{R}^{d}\right)}^{2}+C \delta_{1}\left\|\left(\boldsymbol{u}_{t}, y_{t}\right)\right\|_{H^{2}\left(\mathbb{R}^{d}\right)}^{2} .
$$

Applying integration by parts and the Moser-type calculus (1.12), we can obtain

$$
\begin{aligned}
I_{2} & =\frac{2}{3} \int \partial_{x} \partial_{t}(y \operatorname{div} \boldsymbol{u})\left(\partial_{x} N \partial_{x}^{2} y_{t}+N \partial_{x} \Delta y_{t}\right) \mathrm{d} x \\
& \leqslant C \delta_{1}\left\|\left(\partial_{x} \boldsymbol{u}, \partial_{x} \boldsymbol{u}_{t}, \partial_{x}^{2} \boldsymbol{u}, \partial_{x}^{2} \boldsymbol{u}_{t}, \partial_{x} y_{t}, \partial_{x}^{2} y, \partial_{x}^{2} y_{t}, \partial_{x}^{3} y_{t}\right)\right\|^{2} .
\end{aligned}
$$

Similarly, we can deduce that

$$
\begin{aligned}
& I_{3}=- \frac{2}{3} \int \nabla\left(\frac{\partial_{x}^{2} y}{N+m}\right)\left(N \nabla \partial_{x}^{2} y+\partial_{x}^{2} y \nabla N\right) \mathrm{d} x \\
&+\frac{2}{3} \int\left(\frac{1}{(N+m)^{2}}\left(\partial_{j}(N+m) \Delta \partial_{i} y+\cdots\right) N \partial_{j} \partial_{i} y\right. \\
&\left.\quad+\left(\frac{\partial_{j} \partial_{i}(N+m)}{(N+m)^{2}}-\frac{2 \partial_{j}(N+m) \partial_{i}(N+m)}{(N+m)^{3}}\right) \Delta y N \partial_{j} \partial_{i} y\right) \mathrm{d} x \\
& \leqslant-\int \frac{2 N}{3(N+m)}\left|\nabla \partial_{x}^{2} y\right|^{2} \mathrm{~d} x+C \delta_{1}\left\|\left(\partial_{x}^{2} m, \partial_{x}^{2} y, \nabla \partial_{x}^{2} y\right)\right\|^{2}
\end{aligned}
$$

and

$$
\begin{aligned}
I_{4}=\frac{2}{3} \int( & \frac{\Delta \partial_{x}^{2} y_{t}}{N+m}-\frac{\partial_{i}(N+m) \partial_{j} \Delta y_{t}+\cdots+\partial_{x}^{2}(N+m) \Delta y_{t}}{(N+m)^{2}} \\
& +\frac{2 \partial_{i}(N+m) \partial_{j}(N+m)}{(N+m)^{3}} \Delta y_{t}-\frac{\partial_{x}^{2} m_{t} \Delta y+\partial_{i} m_{t} \partial_{j} \Delta y+\cdots+m_{t} \partial_{x}^{2} \Delta y}{(N+m)^{2}} \\
& +\frac{2 \partial_{i}(N+m)\left(\partial_{j} m_{t} \Delta y+m_{t} \partial_{j} \Delta y\right)}{(N+m)^{3}}+\cdots \\
& \left.+\frac{2 \partial_{x}^{2}(N+m) m_{t} \Delta y}{(N+m)^{3}}-\frac{6 \partial_{i}(N+m) \partial_{j}(N+m) m_{t} \Delta y}{(N+m)^{4}}\right) N \partial_{x}^{2} y_{t} \mathrm{~d} x \\
\leqslant-\int & \frac{2 N}{3(N+m)}\left|\nabla \partial_{x}^{2} y_{t}\right|^{2} \mathrm{~d} x+C \delta_{1}\left\|\left(\partial_{x} m_{t}, \partial_{x}^{2} m, \partial_{x}^{2} m_{t}, \partial_{x}^{2} y, \partial_{x}^{2} y_{t}, \partial_{x}^{3} y, \partial_{x}^{3} y_{t}\right)\right\|^{2} \\
& -\frac{2}{3} \int \frac{1}{(N+m)^{2}}\left(\partial_{x}^{2} m_{t} \Delta y+\partial_{j} m_{t} \partial_{i} \Delta y+\cdots+m_{t} \partial_{x}^{2} \Delta y\right) N \partial_{x}^{2} y_{t} \mathrm{~d} x
\end{aligned}
$$


For the last integral term in (3.62), using integration by parts and the Cauchy-Schwartz inequality (1.11), we have

$$
\begin{aligned}
-\frac{2}{3} \int & \frac{1}{(N+m)^{2}}\left(\partial_{x}^{2} m_{t} \Delta y+\partial_{j} m_{t} \partial_{i} \Delta y+\cdots+m_{t} \partial_{x}^{2} \Delta y\right) N \partial_{x}^{2} y_{t} \mathrm{~d} x \\
=- & \frac{2}{3} \int \frac{1}{(N+m)^{2}} N \partial_{x}^{2} m_{t} \Delta y \partial_{x}^{2} y_{t} \mathrm{~d} x+\frac{2}{3} \int \Delta y \partial_{i}\left(\frac{\partial_{j} m_{t}}{(N+m)^{2}} N \partial_{j} \partial_{i} y_{t}\right) \mathrm{d} x+\cdots \\
\quad & \quad \frac{2}{3} \int \partial_{i} m \partial_{j}\left(\frac{\Delta y_{t}}{(N+m)^{2}} N \partial_{j} \partial_{i} y_{t}\right) \mathrm{d} x \\
\leqslant & C \delta_{1}\left\|\left(\partial_{x} m_{t}, \partial_{x}^{2} m_{t}, \partial_{x}^{2} y_{t}, \partial_{x}^{3} y, \partial_{x}^{3} y_{t}\right)\right\|^{2}
\end{aligned}
$$

Furthermore, (3.58)-(3.63) show that

$$
\begin{aligned}
\frac{1}{2} \frac{\mathrm{d}}{\mathrm{d} t} \int N\left(\left|\partial_{x}^{2} y\right|^{2}+\right. & \left.\left|\partial_{x}^{2} y_{t}\right|^{2}\right) \mathrm{d} x+C\left\|\left(\partial_{x}^{3} y, \partial_{x}^{3} y_{t}\right)\right\|^{2}+\frac{2}{3} T^{(0)} \int N \partial_{x}^{2} y_{t} \partial_{x}^{2} \operatorname{div} \boldsymbol{u}_{t} \mathrm{~d} x \\
& \leqslant C\|(m, \boldsymbol{u}, y)\|_{H^{2}\left(\mathbb{R}^{d}\right)}^{2}+C \delta_{1}\left\|\left(\partial_{x} m_{t}, \partial_{x}^{2} m_{t}, \partial_{x} \boldsymbol{u}_{t}, \partial_{x}^{2} \boldsymbol{u}_{t}, \partial_{x}^{2} y_{t}\right)\right\|^{2}
\end{aligned}
$$

Combining (3.57) and (3.64), one obtains

$$
\begin{aligned}
& \frac{\mathrm{d}}{\mathrm{d} t} \int\left(\left|\partial_{x}^{2} \boldsymbol{u}_{t}\right|^{2}\right.+\frac{2}{N}\left(\partial_{i} N \partial_{j} \boldsymbol{u}_{t} \partial_{x}^{2} \boldsymbol{u}_{t}+\cdots+\partial_{x}^{2} N \partial_{x}^{2} \boldsymbol{u}_{t} \boldsymbol{u}_{t}\right) \\
&\left.+\frac{T^{(0)}+y}{(N+m)^{2}}\left|\partial_{x}^{2} m_{t}\right|^{2}+\frac{1}{N}\left|\partial_{x}^{2} \varphi_{t}\right|^{2}+\frac{3}{2 T^{(0)}}\left(\left|\partial_{x}^{2} y\right|^{2}+\left|\partial_{x}^{2} y_{t}\right|^{2}\right)\right) \mathrm{d} x+C\left\|\left(\partial_{x}^{2} \boldsymbol{u}_{t}, \partial_{x}^{2} \varphi_{t}, \partial_{x}^{3} y, \partial_{x}^{3} y_{t}\right)\right\|^{2} \\
&+C\|(m, \boldsymbol{u}, \varphi, y)\|_{H^{2}\left(\mathbb{R}^{d}\right)}^{2} \\
&+C \delta_{1}\left(\left\|\left(m_{t}, \boldsymbol{u}_{t}, \varphi_{t}, y_{t}\right)\right\|_{H^{2}\left(\mathbb{R}^{d}\right)}^{2}+\left\|\left(\partial_{x}^{3} m, \partial_{x}^{3} \boldsymbol{u}, \partial_{x}^{3} \varphi\right)\right\|^{2}\right) .
\end{aligned}
$$

Therefore, combining (3.56), (3.55), (3.65) and (3.8) with $i=2$ and (3.9) with $j=3$, we obtain

$$
\begin{gathered}
\frac{\mathrm{d}}{\mathrm{d} t} \int\left(\left|\partial_{x}^{3} \boldsymbol{u}\right|^{2}+\frac{T^{(0)}+y}{(N+m)^{2}}\left|\partial_{x}^{3} m\right|^{2}+\frac{2}{N}\left(\partial_{h} \partial_{i} N \partial_{j} \boldsymbol{u} \partial_{x}^{3} \boldsymbol{u}+\cdots+\partial_{h} N \partial_{x}^{3} \boldsymbol{u} \partial_{i} \partial_{j} \boldsymbol{u}\right.\right. \\
\left.+\cdots+\boldsymbol{u} \partial_{x}^{3} N \partial_{x}^{3} \boldsymbol{u}\right)+\left|\partial_{x}^{2} \boldsymbol{u}_{t}\right|^{2}+\frac{2}{N}\left(\partial_{i} N \partial_{j} \boldsymbol{u}_{t} \partial_{x}^{2} \boldsymbol{u}_{t}+\cdots+\boldsymbol{u}_{t} \partial_{x}^{2} N \partial_{x}^{2} \boldsymbol{u}_{t}\right) \\
\left.+\frac{T^{(0)}+y}{(N+m)^{2}}\left|\partial_{x}^{2} m_{t}\right|^{2}+\frac{1}{N}\left(\left|\partial_{x}^{3} \varphi\right|^{2}+\left|\partial_{x}^{2} \varphi_{t}\right|^{2}\right)+\frac{3}{2 T^{(0)}}\left(\left|\partial_{x}^{2} y\right|^{2}+\left|\partial_{x}^{2} y_{t}\right|^{2}\right)\right) \mathrm{d} x \\
+C\left\|\left(\partial_{x}^{2} m_{t}, \partial_{x}^{3} m, \partial_{x}^{2} u_{t}, \partial_{x}^{3} \boldsymbol{u}, \partial_{x}^{2} \varphi_{t}, \partial_{x}^{3} \varphi, \partial_{x}^{2} y, \partial_{x}^{2} y_{t}, \partial_{x}^{3} y, \partial_{x}^{3} y_{t}\right)\right\|^{2} \\
\leqslant C\left(\left\|\left(m, \boldsymbol{u}, \varphi, y, y_{t}\right)\right\|_{H^{2}\left(\mathbb{R}^{d}\right)}^{2}+C \delta_{1}\left\|\left(m_{t}, \boldsymbol{u}_{t}, \varphi_{t}, y_{t}\right)\right\|_{H^{2}\left(\mathbb{R}^{d}\right)}^{2}\right)
\end{gathered}
$$

Finally, summing up (3.41), (3.46) and (3.66), we find that

$$
\frac{\mathrm{d}}{\mathrm{d} t} \int G \mathrm{~d} x+C\|(m, \boldsymbol{u}, \varphi, y)\|_{H^{3}\left(\mathbb{R}^{d}\right)}^{2}+C\left\|\left(m_{t}, \boldsymbol{u}_{t}, y_{t}, \varphi_{t}\right)\right\|_{H^{2}\left(\mathbb{R}^{d}\right)}^{2} \leqslant 0,
$$


where

$$
\begin{aligned}
G=A_{2}\left\{A _ { 1 } \left[|\boldsymbol{u}|^{2}+\left|\boldsymbol{u}_{t}\right|^{2}+\frac{T^{(0)}+y}{(N+m)^{2}}\left(m^{2}+m_{t}^{2}\right)+\frac{1}{N}\left(|\varphi|^{2}+\left|\varphi_{t}\right|^{2}\right)+\lambda_{1}|\nabla \varphi|^{2}\right.\right. \\
\left.\quad+\eta_{1}\left(|\nabla \boldsymbol{u}|^{2}+\frac{1}{N} m^{2}+\frac{T^{(0)}+y}{(N+m)^{2}}|\nabla m|^{2}\right)+\frac{3}{2 T^{(0)}}\left(y^{2}+y_{t}^{2}\right)\right] \\
+\eta_{2}\left(\left|\partial_{x}^{2} \boldsymbol{u}\right|^{2}+\frac{2}{N}\left(\boldsymbol{u} \partial_{x}^{2} N \partial_{x}^{2} \boldsymbol{u}+\partial_{i} N \partial_{j} \boldsymbol{u} \partial_{x}^{2} \boldsymbol{u}+\cdots\right)\right. \\
\left.\quad+\frac{1}{N}\left|\partial_{x}^{2} \varphi\right|^{2}+\frac{T^{(0)}+y}{(N+m)^{2}}\left|\partial_{x}^{2} m\right|^{2}\right) \\
+\left|\partial_{x} \boldsymbol{u}_{t}\right|^{2}+\frac{T^{(0)}+y}{(N+m)^{2}}\left|\partial_{x} m_{t}\right|^{2}+\frac{1}{N}\left|\partial_{x} \varphi_{t}\right|^{2} \\
\left.\quad+\frac{2}{N} \boldsymbol{u}_{t} \partial_{x} N \partial_{x} \boldsymbol{u}_{t}+\frac{3}{2 T^{(0)}}\left(\left|\partial_{x} y\right|^{2}+\left|\partial_{x} y_{t}\right|^{2}\right)\right\} \\
+\left|\partial_{x}^{3} \boldsymbol{u}\right|^{2}+\left|\partial_{x}^{2} \boldsymbol{u}_{t}\right|^{2}+\frac{1}{N}\left(\left|\partial_{x}^{3} \varphi\right|^{2}+\left|\partial_{x}^{2} \varphi_{t}\right|^{2}\right)+\frac{T^{(0)}+y}{(N+m)^{2}}\left(\left|\partial_{x}^{3} m\right|^{2}+\left|\partial_{x}^{2} m_{t}\right|^{2}\right) \\
+\frac{2}{N}\left(\boldsymbol{u} \partial_{x}^{3} N \partial_{x}^{3} \boldsymbol{u}+\partial_{h} \partial_{i} N \partial_{j} \boldsymbol{u} \partial_{x}^{3} \boldsymbol{u}+\cdots+\partial_{h} N \partial_{i} \partial_{j} \boldsymbol{u} \partial_{x}^{3} \boldsymbol{u}+\cdots\right. \\
\left.\quad+\partial_{i} N \partial_{j} \boldsymbol{u}_{t} \partial_{x}^{2} \boldsymbol{u}_{t}+\cdots+\partial_{x}^{2} N \boldsymbol{u}_{t} \partial_{x}^{2} \boldsymbol{u}_{t}\right) \\
+\frac{3}{2 T^{(0)}}\left(\left|\partial_{x}^{2} y\right|^{2}+\left|\partial_{x}^{2} y_{t}\right|^{2}\right), \quad
\end{aligned}
$$

for some positive constants $A_{1}$ and $A_{2}$.

It is easy to verify that $G$ satisfies

$$
\begin{aligned}
& a\left(\|(m, \boldsymbol{u}, \varphi)\|_{H^{3}\left(\mathbb{R}^{d}\right)}^{2}+\|y\|_{H^{2}\left(\mathbb{R}^{d}\right)}^{2}+\left\|\left(m_{t}, \boldsymbol{u}_{t}, \varphi_{t}, y_{t}\right)\right\|_{H^{2}\left(\mathbb{R}^{d}\right)}^{2}\right) \\
& \quad \leqslant \int G \mathrm{~d} x \leqslant b\left(\|(m, \boldsymbol{u}, \varphi)\|_{H^{3}\left(\mathbb{R}^{d}\right)}^{2}+\|y\|_{H^{2}\left(\mathbb{R}^{d}\right)}^{2}+\left\|\left(m_{t}, \boldsymbol{u}_{t}, \varphi_{t}, y_{t}\right)\right\|_{H^{2}\left(\mathbb{R}^{d}\right)}^{2}\right),
\end{aligned}
$$

for some positive constants $b>a>0$ and $A_{1}, A_{2}>0$.

Hence, the use of Gronwall's inequality, (3.67) and (3.68) implies (3.3) for any $t \in(0, S]$, and Lemma 3.3 is proved.

Acknowledgements. I express my deep thanks to my advisor, Professor Zhouping Xin, whose support and encouragement have been necessary for the completion of this work. I also thank the referees for their comments and suggestions.

\section{References}

1. G. Ali, Global existence of smooth solutions of the $N$-dimensional Euler-Poisson model, J. Diff. Eqns 190(2) (2003), 663-685.

2. G. Ali, P. Marcati and R. NAtalini, Hydrodynamical models for semiconductors, $Z$. Angew. Math. Mech. 76 (1996), 301-304.

3. G. Ali, D. Bini And S. Rionero, Global existence and relaxation limit for smooth solutions to the Euler-Poisson model for semiconductors, SIAM J. Math. Analysis 32(3) (2000), 572-587. 
4. A. M. ANILE, An extended thermodynamic framework for the hydrodynamical modelling of semiconductors, in Mathematical problems in semiconductor physics (ed. P. Marcati, P. A. Markowich and R. Natalini), vol. 3-41, Pitman Research Notes in Mathematics, vol. 340 (Longman, Harlow, 1995).

5. A. M. Anile And O. Muscato, Improved hydrodynamical model from carrier transport in semiconductors, Phys. Rev. B 51 (1995), 16 728-16 740.

6. A. M. Anile And S. PEnnisi, Extended thermodynamics of the Blotekjaer hydrodynamical model for semiconductors, Continuum Mech. Thermodyn. 4 (1992), 187-197.

7. P. Degond And P. A. Markowich, On a one-dimensional steady-state hydrodynamic model for semiconductors, Appl. Math. Lett. 3 (1990), 25-29.

8. P. DEgond, S. Genieys AND A. Jüngel, A steady-state system in nonequilibrium thermodynamics including thermal and electrical effects, C. R. Acad. Sci. Paris Sér. I 324 (1997), 867-872.

9. W. FANG AND K. ITO, Weak solutions to a one-dimensional hydrodynamic model for semiconductors, Nonlin. Analysis 28 (1997), 947-963.

10. I. M. GAmbA, Stationary transonic solutions of a one-dimensional hydrodynamic model for semiconductors, Commun. PDEs 17 (1992), 553-577.

11. I. GASSER AND R. NATALini, The energy transport and the drift diffusion equations as relaxation limits of the hydrodynamic model for semiconductors, Q. Appl. Math. $\mathbf{5 7}$ (1999), 269-282.

12. Y. GuO, Smooth irrotational fluids in the large to the Euler-Poisson system in $\mathbb{R}^{3+1}$, Commun. Math. Phys. 195 (1998), 249-265.

13. L. HSiao AND S. WAng, Asymptotic behavior of global smooth solutions to the full 1D hydrodynamic model for semiconductors, Math. Models Meth. Appl. Sci. 12 (2002), 777-796.

14. L. HSiao And T. YAnG, Asymptotics of initial boundary value problems for hydrodynamic and drift diffusion models for semiconductors, J. Diff. Eqns 170 (2001), 472-493.

15. L. Hsiao, P. A. MARKowich AND S. WANG, The asymptotic behavior of globally smooth solutions of the multidimensional isentropic hydrodynamic model for semiconductors, $J$. Diff. Eqns 192(1) (2003), 111-133.

16. F. Jochmann, Global weak solutions of the one-dimensional hydrodynamic model for semiconductors to the drift-diffusion equations, Math. Models Meth. Appl. Sci. 3 (1993), 759-788.

17. A. JüngeL, Quasi-hydrodynamic semiconductor equations, Progress in Nonlinear Differential Equations, Vol. 41 (Birkhäuser, 2001).

18. T. Luo, R. NAtalini And Z. Xin, Large time behavior of the solutions to a hydrodynamic model for semiconductors, SIAM J. Appl. Math. 59(3) (1998), 810-830.

19. A. MAJDA, Compressible fluid flow and systems of conservation laws in several space variables, Applied Mathematical Sciences, vol. 53 (Springer, 1984).

20. P. MARCATi AND R. NATAlini, Weak solutions to a hydrodynamic model for semiconductors and relaxation to the drift-diffusion equation, Arch. Ration. Mech. Analysis 129 (1995), 129-145.

21. P. A. Markowich, On steady-state Euler-Poisson model for semiconductors, Z. Angew. Math. Phys. 62 (1991), 389-407.

22. P. A. Markowich, C. A. Ringhofev And C. Schmeiser, Semiconductors equations (Spring, 1990).

23. A. Matsumura And T. Nishida, The initial value problem for the equations of motion of viscous and heat-conductive gases, J. Math. Kyoto Univ. 20(1) (1980), 67-104.

24. L. Nirenberg, On elliptic partial differential equations, Annli Scuola Norm. Sup. Pisa 13 (1959), 115-162.

25. F. Poupaud, M. Rascle And J. P. Vila, Global solutions to the isothermal EulerPoisson system with arbitrarily large data, J. Diff. Eqns 123 (1995), 93-121. 
26. E. M. StEIn, Singular integrals and differentiability properties of functions (Princeton University Press, Princeton, NJ, 1970).

27. B. Zhang, Convergence of the Godunov scheme for a simplified one-dimensional hydrodynamic model for semiconductors, Commun. Math. Phys. 157 (1993), 1-22. 